\title{
CLASSICAL T TAURI-LIKE OUTFLOW ACTIVITY IN THE BROWN DWARF MASS REGIME*
}

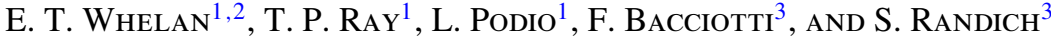 \\ ${ }^{1}$ School of Cosmic Physics, Dublin Institute for Advanced Studies, Republic of Ireland \\ ${ }^{2}$ Laboratoire d'Astrophysique de l'Observatoire de Grenoble, BP 53, F-38041 Grenoble cedex, France \\ ${ }^{3}$ INAF/Osservatorio Astrofisico di Arcetri, Largo Enrico Fermi 5, I-50125 Firenze, Italia \\ Received 2009 August 15; accepted 2009 October 16; published 2009 November 9
}

\begin{abstract}
Over the last number of years, spectroscopic studies have strongly supported the assertion that protostellar accretion and outflow activity persist to the lowest masses. Indeed, previous to this work, the existence of three brown dwarf (BD) outflows had been confirmed by us. In this paper, we present the results of our latest investigation of BD outflow activity and report on the discovery of two new outflows. Observations to date have concentrated on studying the forbidden emission line (FEL) regions of young BDs and in all cases data have been collected using the UV-Visual Echelle Spectrometer (UVES) on the ESO Very Large Telescope. Offsets in the FEL regions are recovered using spectro-astrometry. Here, ISO-Oph 32 is shown to drive a blueshifted outflow with a radial velocity of $10-20 \mathrm{~km} \mathrm{~s}^{-1}$ and spectro-astrometric analysis constrains the position angle of this outflow to $240^{\circ} \pm 7^{\circ}$. The $\mathrm{BD}$ candidate, ISO-ChaI 217 is found to have a bipolar outflow bright in several key forbidden lines $\left(\mathrm{V}_{\mathrm{RAD}}=\right.$ $-20 \mathrm{~km} \mathrm{~s}^{-1},+40 \mathrm{~km} \mathrm{~s}^{-1}$ ) and with a P.A. of $193^{\circ}-209^{\circ}$. A striking feature of the ISO-ChaI 217 outflow is the strong asymmetry between the red- and blueshifted lobes. This asymmetry is revealed in the relative brightness of the two lobes (redshifted lobe is brighter), the factor of 2 difference in radial velocity (the redshifted lobe is faster) and the difference in the electron density (again higher in the red lobe). Such asymmetries are common in jets from low-mass protostars and the observation of a marked asymmetry at such a low mass $\left(<0.1 M_{\odot}\right)$ supports the idea that BD outflow activity is scaled down from low-mass protostellar activity. Also note that although asymmetries are unexceptional, it is uncommon for the redshifted lobe to be the brightest as some obscuration by the accretion disk is assumed. This phenomenon has only been observed in one other source, the classical T Tauri (CTTS) star RW Aur. The physical mechanism responsible for the brightening of the redshifted lobe although as yet unknown must also now apply at BD masses to include the ISO-ChaI 217 outflow. In addition to presenting these new results, a comprehensive comparison is made between BD outflow activity and jets launched by CTTSs. In particular, the application of current methods for investigating the excitation conditions and mass loss rates $\left(\dot{M}_{\text {out }}\right)$ in CTT jets to BD spectra is explored. Where possible, the Bacciotti \& Eislöffel technique is used to study the ionization fraction, electron temperature, and total density. For LS-RCrA 1 , ISO-ChaI 217 and ISO-Oph $102 \dot{M}_{\text {out }}$ are measured to be in the range $10^{-10}$ to $10^{-9} M_{\odot} \mathrm{yr}^{-1}$ using a method based on the luminosity of the $\left[\mathrm{O}_{\mathrm{I}}\right] \lambda 6300$ and $\left[\mathrm{S}_{\mathrm{II}}\right] \lambda 6731$ lines. Mass loss rates for our sample of BD outflows are found to be comparable to the mass accretion rates. Overall, as our results and discussion show, what is currently known about outflow activity in the BD mass regime points to strong similarities between BD outflows and CTT jets.
\end{abstract}

Key words: ISM: jets and outflows - stars: formation - stars: low-mass, brown dwarfs

\section{INTRODUCTION}

The outflow phenomenon, much studied in young stars has been observed in a wide variety of astrophysical objects from low-mass protostars to active galactic nuclei (AGNs; Reipurth $\&$ Bally 2001; Camenzind 2005). Brown Dwarfs (BDs) are the newest objects to be added to this list (Fernández \& Comerón 2001; Barrado y Navascués et al. 2004; Whelan et al. 2005, 2007; Phan-Bao et al. 2008; Whelan et al. 2009). We are currently leading a project to search for and study outflows driven by young BDs and very low mass stars. The overall aim of this work is to explore outflow activity in the BD mass regime through comparison with observations of outflows driven by low-mass protostars and the classical T Tauri stars (CTTSs) in particular. Much emphasis has been placed on understanding how accretion activity (Gatti et al. 2006; Herczeg et al. 2009) and planet formation processes (Pascucci et al. 2009) differ between low-mass protostars and young BDs. However, little is still known about whether the outflow mechanism varies as

\footnotetext{
* Based on data collected by UVES observations (079.C-0375(A)) at the VLT on Cerro Paranal (Chile) which is operated by the European Southern Observatory (ESO).
}

mass decreases toward the BD regime. Observations aimed at detecting outflows from such low-mass objects are difficult and rare and to date six candidates have been investigated by us spectroscopically at visible wavelengths.

Outflows from low-mass protostars are revealed in several forms, from slow wide angled winds to molecular outflows and jets (Reipurth \& Bally 2001). A protostellar jet is a relatively fast (typical radial velocity $=200 \mathrm{~km} \mathrm{~s}^{-1}$ ) collimated outflow normally seen within a few $1000 \mathrm{AU}$ of a young star and bright in optical and/or near-infrared shock tracers such as [S II] $]$ 6731, [Fe II] at $1.644 \mu \mathrm{m}$ or $\mathrm{H}_{2}$ at $2.122 \mu \mathrm{m}$. CTTSs are Class II low-mass protostars (Lada 1987; Andre et al. 1993), meaning that they are coming toward the end of their accretion/outflow phase and have crossed the so-called birth-line (Stahler 1983). As they are optically visible, the jet can be traced very close to the protostar, hence, they offer a unique opportunity to study the launching of protostellar jets at high spatial resolution (Ray et al. 2007). Jets from CTTSs are traditionally probed at forbidden emission line (FEL) wavelengths and long-slit and integral field spectroscopic techniques have been hugely important in their study (Dougados et al. 2000; Whelan et al. 2004). That CTTlike outflows could be launched by actively accreting BDs was 
Table 1

Spectral Type and Predicted Mass of the BD Candidates Investigated by Us to Date

\begin{tabular}{lcccc}
\hline \hline \multicolumn{1}{c}{ Source } & R.A. (J2000) & Decl. (J2000) & Spectral Type & Mass $\left(M_{\text {JUP }}\right)$ \\
\hline ISO-ChaI 217 & 110952.0 & -763912.0 & M6.2 & $80^{1}$ \\
2MASS1207-3932 & 120733.4 & -393254.0 & M8 & $24^{2}$ \\
DENIS-P J160603.9-205644 & 160603.90 & -205644.6 & M7.5 & $40^{3}$ \\
ISO-Oph 32 & 162622.05 & -244437.5 & M8 & $40^{4}$ \\
ISO-Oph 102 & 162706.58 & -244147.9 & M6 & $60^{4}$ \\
LS-RCrA 1 & 190133.7 & -370030.0 & M6.5 & $35-72^{5}$ \\
\hline
\end{tabular}

Notes. All sources except DENIS-P J160603.9-205644 are found to drive outflows. The numbers 1-6 refer to the papers giving the estimates of the mass of each source, where 1, Muzerolle et al. (2005); 2, Mohanty et al. (2007); 3, Mohanty et al. (2004); 4, Natta et al. (2002); and 5, Barrado y Navascués et al. (2004).

first suggested when high-quality spectra revealed the presence of FEL regions in the optical spectra of BDs known to be accretors (Fernández \& Comerón 2001). While the FEL regions were easily identified, they were considerably fainter than those detected in the spectra of CTTSs (Masciadri \& Raga 2004) and any extension in the form of an outflow was not apparent (Fernández \& Comerón 2001, 2005). Hence, their origin in an outflow could not be established. The critical density region for FELs occurs much closer to the driving source of the outflow for nearby $(\sim 150 \mathrm{pc})$ young BDs than for low-mass protostars and for BDs is estimated to be on scales of $\sim 100$ mas (Whelan et al. 2005). As the most intense forbidden emission coincides with the critical density region it is currently challenging to directly resolve a BD outflow in FELs and this goal has not yet been achieved. Our approach to this problem has been to obtain high-quality spectra using the UV-Visual Echelle Spectrometer (UVES) on the European Southern Observatory's (ESO) Very Large Telescope (VLT) and to recover the spatial offset in the region of forbidden emission using spectro-astrometry.

The sources targeted so far meet at least one of two criteria. Firstly, they all have masses at or significantly below the hydrogen burning mass limit (HBML) and second they are known to be powerful accretors. Their classification as a strong accretor is based on studies of their $\mathrm{H} \alpha$ lines (Jayawardhana et al. 2003b) and on measurements of their mass accretion rates (see Table 6). In the vast majority of cases, the mass of young BD candidates cannot be directly measured and estimates are based on theoretical predictions (Luhman et al. 2000) and/or spectral modeling (Mohanty et al. 2004). The objects investigated by us so far are listed in Table 1 along with their theoretical masses and spectral types. Previous to this paper, we have reported on the discovery of and discussed the optical outflows from, ISOOph 102 (Whelan et al. 2005), 2MASS1207-3932 (Whelan et al. 2007), and LS-RCrA 1 (Whelan et al. 2009). With a mass of only $24 M_{\mathrm{JUP}}, 2 \mathrm{MASS} 1207-3932$ is at present the lowest mass galactic object driving an outflow. In this paper, we present the results of our recent observations of ISO-ChaI 217, DENIS-P J160603.9-205644, and ISO-Oph 32 and report for the first time outflows driven by ISO-ChaI 217 and ISO-Oph 32. Of the six objects observed by us to date only one, ISO-ChaI 217 has a theoretical mass which is just above the HBML, placing it at present just outside the mass range for a BD. However, with a predicted mass of just $80 M_{\mathrm{JUP}}$, it is still one of the lowest mass objects known to launch an outflow. Therefore, for the purpose of the discussion we will include it in this paper with the likely BDs. In addition to presenting our spectro-astrometric analysis, a technique for constraining the position angle (P.A.) of the BD outflows is outlined, methods for studying the physical conditions of the BD outflow and of estimating the mass outflow rate are discussed, and similarities between BD outflows and CTT jets highlighted and considered.

\section{OBSERVATIONS AND SPECTRO-ASTROMETRIC ANALYSIS}

Spectra (at orthogonal slit positions) of ISO-Cha1 217, ISOOph 32, and DENIS-P J160603.9-205644 were obtained with the high resolution spectrometer UVES (CD3 disperser, spectral range 4810-6740 $, R=40,000$ ), on the ESO VLT UT2 (Dekker et al. 2000), as part of the observing program 079.C-0375(A). In each case, the exposure time was $2700 \mathrm{~s}$, the slit width $1^{\prime \prime}$ and the seeing varied between 0.5 and $0{ }^{\prime} .9$. Spectra were analyzed using spectro-astrometry as outlined in previous papers (Whelan et al. 2005, 2007). Also see Whelan \& Garcia (2008) for details on basic data reduction steps and continuum/night sky line subtraction. To summarize, spectro-astrometry involves the measurement of the spatial centroid of an emission line (using Gaussian fitting) as a function of velocity. The centroid of the continuum emission is taken as the position of the star/BD and the emission line centroid is measured with respect to this position.

Problems specific to the application of spectro-astrometry to BD spectra are the lack of or weakness of continuum emission from the BD in the range 5000-8000 $\AA$ and the faintness of the line emission itself. In order to overcome these problems and increase the spectro-astrometric accuracy, the spectra are summed or smoothed in the dispersion direction. In the absence of detector noise, the spectro-astrometric accuracy is dependent on $N_{p}^{1 / 2}$ where $N_{p}$ is the number of detected photons in the line/ continuum (Whelan et al. 2005). In the cases, where spectra are summed, the line and continuum are summed separately and by differing amounts, so that the spectro-astrometric error in the line and continuum position is comparable. Recall that the continuum must be removed before the line centroid is fitted. In these cases, points are a moving sum across the line and continuum (see results for ISO-Cha 1217 and DENIS-P J160603.9-205644). For BDs where the line emission is weaker than the underlying continuum emission it was more effective to decrease the spectral resolution and hence increase the signal by smoothing the spectrum using a Gaussian filter (Whelan et al. 2007). Again this is done so that errors are comparable. The $\left[\mathrm{O}_{\mathrm{I}}\right] \lambda 6300$ line region in the spectra of ISO-Oph 32 was smoothed using a Gaussian filters of FWHM $=0.2 \AA \times$ 0.32 . In all cases, the dashed line delineates the $1 \sigma$ error in the continuum (and hence line) centroid. Finally, the issue of spectro-astrometric artifacts must be addressed. Spectroastrometric artifacts, or false spectro-astrometric signals, can 
<smiles>NCF</smiles>

UVES slit at P.A. $=0$ degrees

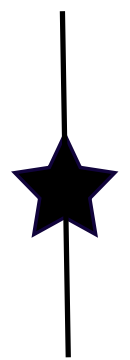

+ve offset, emission extended in northerly direction

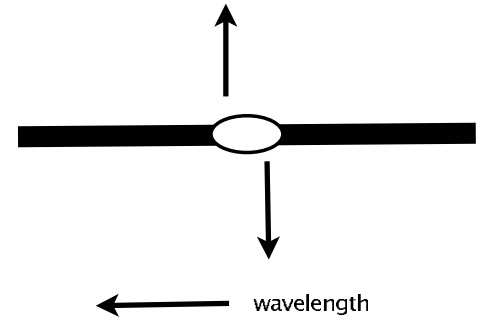

UVES slit at P.A. $=90$ degrees

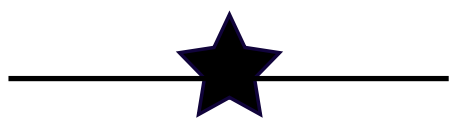

+ve offset, emission extended in easterly direction

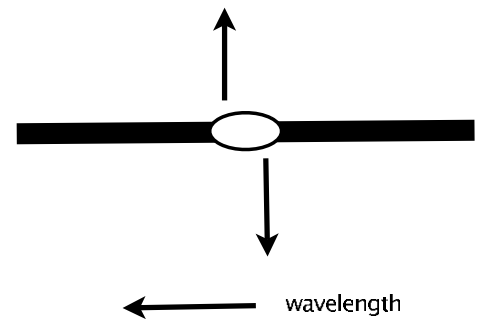

-ve offset, emission extended in westerly direction

-ve offset, emission extended in southerly direction

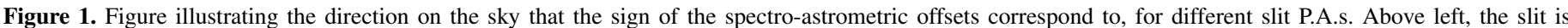

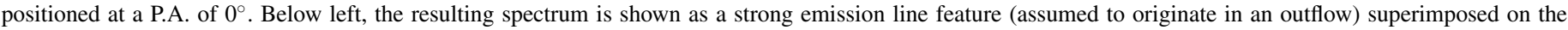

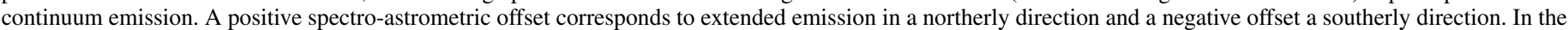
case of an E-W slit position (P.A. $=90^{\circ}$ ), a positive offset corresponds to an easterly direction and negative to a westerly. Wavelength increases toward the left.

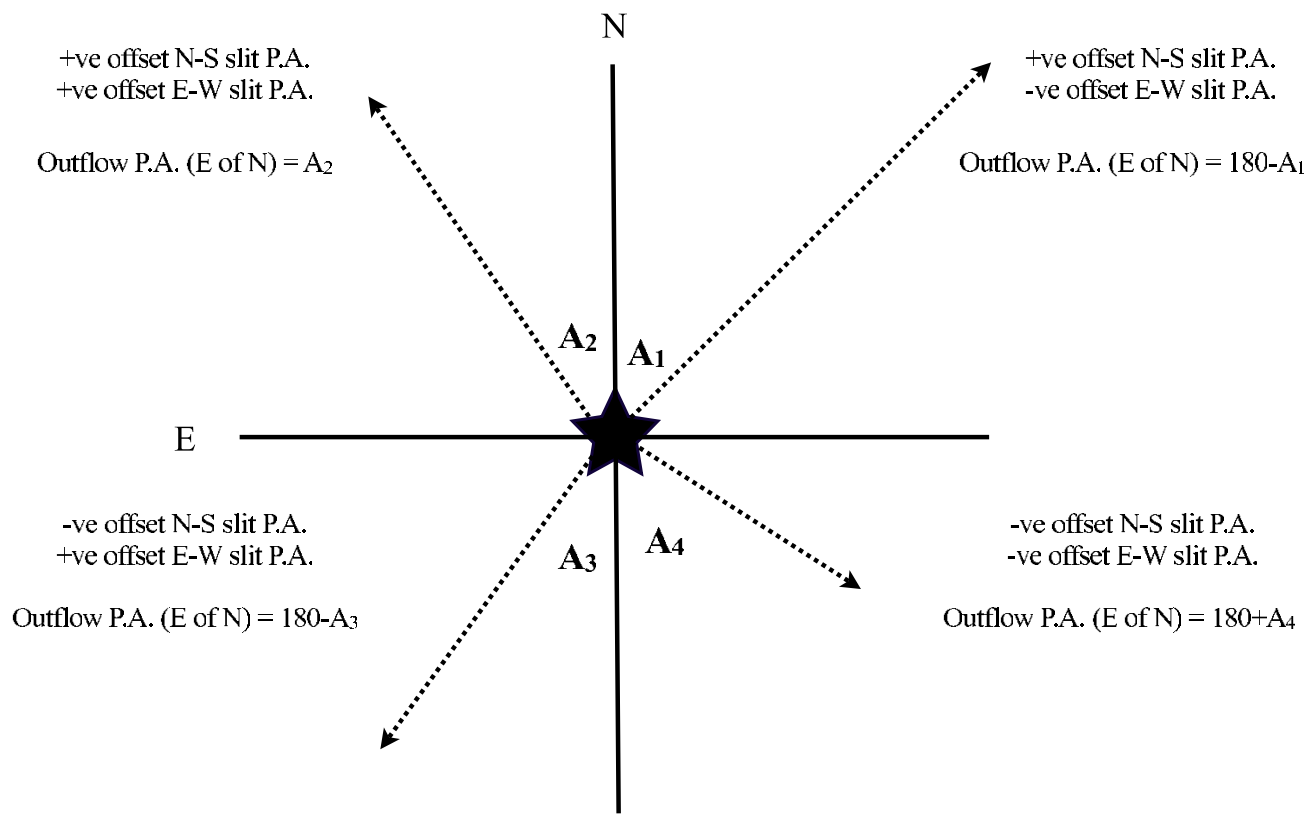

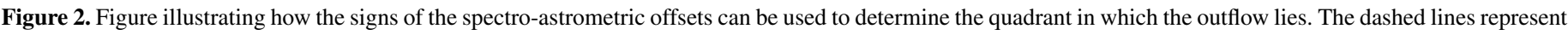

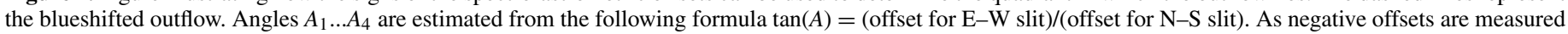
for both the ISO-ChaI 217 and ISO-Oph 32 outflows, the blueshifted lobes lie in the fourth quadrant.

be introduced into a spectrum for a variety of reasons (Podio et al. 2008). In our studies of BD outflows, we have ruled out the influence of such artifacts by demonstrating that lines like HeI $\lambda 6678$, which will not form in an outflow, do not have any spectro-astrometric signal. If the outflow signatures detected in the FELs were due to artifacts then these lines would also show similar offsets. In addition, note that the $\mathrm{H} \alpha$ line in the majority of cases does not show any offset. This is in agreement with the idea that most of the $\mathrm{H} \alpha$ emission comes from the accretion zone close to the source. For all the BDs, spectra are 

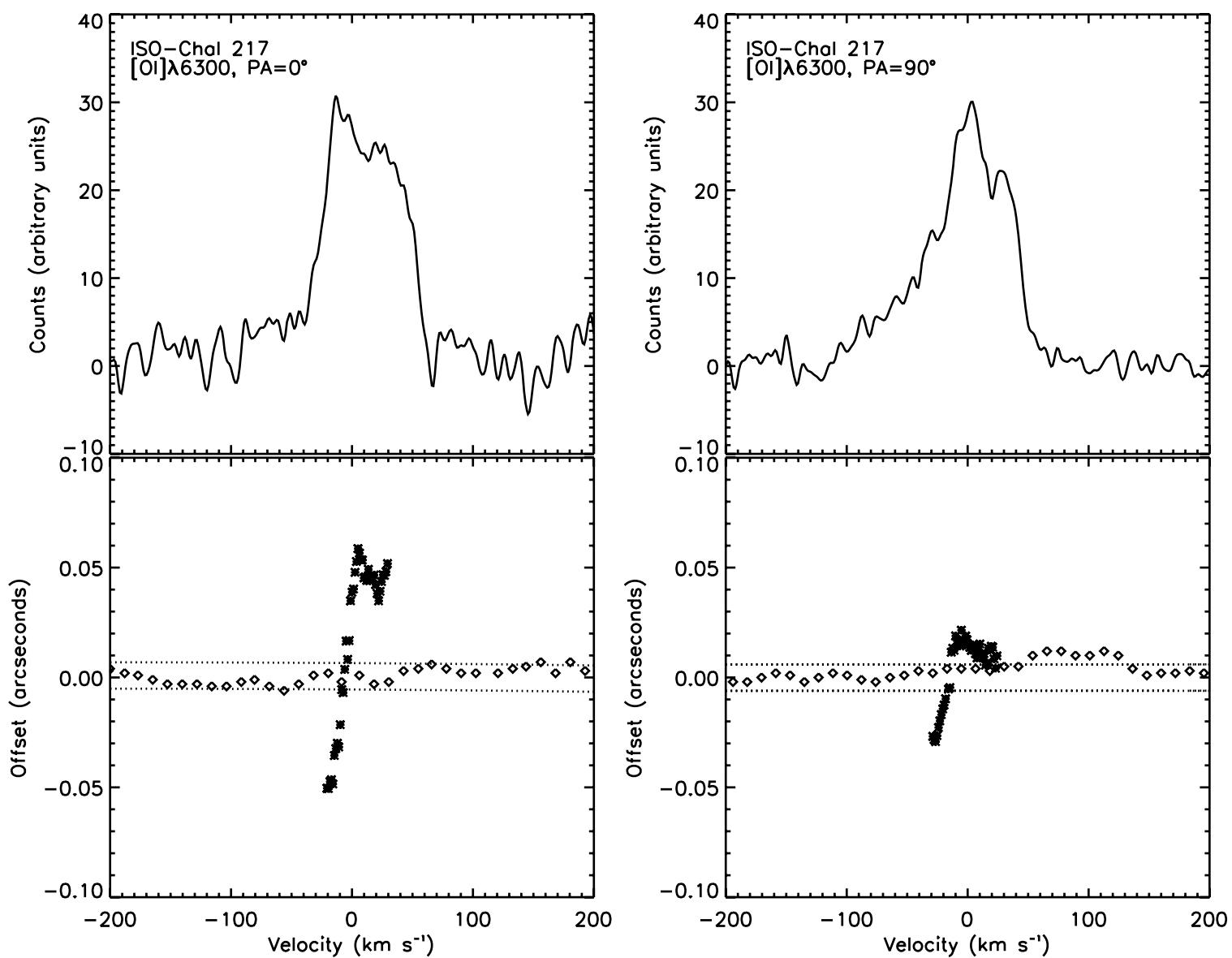

Figure 3. Spectro-astrometric analysis of the ISO-Cha2 17 [O I] $\lambda 6300$ line. The line has a blue and a redshifted component. In the spectrum taken at $90^{\circ}$, the blueshifted component to the line appears as an extended wing. Offsets decrease at $90^{\circ}$ and comparison with measurements at $0^{\circ}$ constrain the outflow P.A. at $\sim 208^{\circ}$. Offsets are a moving sum across the line and continuum position. The displacement in the line is measured in the continuum subtracted spectrum. The $1 \sigma$ error in the line is marked by the dashed line. Finally, as outlined in Section 2 the line and continuum emission is summed in such a way that the $1 \sigma$ error in both is comparable.

presented at several P.A.s, and offsets measured at the different P.A.s agree. Lastly, the position velocity (PV) diagram of the emission line regions of ISO-ChaI 217 supports the spectroastrometric analysis.

\section{RESULTS OF SPECTRO-ASTROMETRIC ANALYSIS}

In this section, the results of the spectro-astrometric analysis of the $\mathrm{FE}$ and $\mathrm{H} \alpha$ line regions of, ISO-Cha1 217, ISO-Oph 32, and DENIS-P J160603.9-205644, are presented. The particulars of the spectro-astrometric technique, as applied to $\mathrm{BD}$ spectra, are discussed above. As well as recovering the spatial offset in the FEL regions of our BD candidates (FELs were only detected in the case of ISO-ChaI 217 and ISO-Oph 32), this technique is also used to estimate the P.A. of the new BD outflows, through comparison of the spectro-astrometric offsets measured at orthogonal slit positions. Jets driven by CTTSs were first observed using narrow band imagery and long-slit spectroscopy (Mundt \& Fried 1983; Solf \& Boehm 1993). Hirth et al. (1994, 1997) estimated the micro-jet P.A. for a sample of CTTSs by taking spectra at several slit P.A.s and plotting the positional offset and spatial full width at half-maximum (FWHM) of the [S II] $]$ 6731 line as a function of slit P.A. They argue that the $[\mathrm{S} \mathrm{II}] \lambda 6731$ line is a better indicator of the outflow direction than the [O I] $\lambda 6300$ line, as its lower critical density means that it traces the jet at a greater distance from the source. Closer to the source, other outflow components e.g., the low-velocity component could influence any measurement of the jet P.A. Although the approach of Hirth et al. (1994) would provide a more straightforward way of estimating the outflow P.A. for our BD candidates, the number of spectra that could be collected was limited by the faintness of the FELs and thus the on source time needed to detect them with a reasonable signal-to-noise ratio ( $\mathrm{S} / \mathrm{N}$; on average 45 minutes with the VLT). For UVES, a negative spectro-astrometric offset, at a $\mathrm{N}-\mathrm{S}$ slit P.A. equals a southerly displacement in an emission feature and a positive offset a northerly displacement. For an E-W slit P.A., negative and positive offsets correspond to westerly and easterly directions, respectively. This is illustrated in Figure 1, comparison of the signs of the $\mathrm{N}-\mathrm{S}, \mathrm{E}-\mathrm{W}$ spectro-astrometric offsets allows the quadrant in which the blueshifted outflow lies to be ascertained. Once this is known, the actual outflow P.A. (E of N) can be derived. In Figure 2, the dashed, arrowed lines represent the blueshifted lobe of the outflow as located in each quadrant. The angles $A_{1}$ to $A_{4}$ are simply estimated from $\tan \left(A_{1 \ldots 4}\right)=$ (offset for $\mathrm{E}-\mathrm{W}$ slit $) /$ (offset for N-S slit).

Error is introduced by the fact that the positional offset in the FEL regions is not directly resolved but recovered using spectro-astrometry. The accuracy with which one can measure the positional displacement of an unresolved emission feature using Gaussian fitting depends primarily on the $\mathrm{S} / \mathrm{N}$ of the emission feature. However, the displacement is also weighted by the uniformity of the emission. One emission feature could actually be made up of several unresolved features of differing 

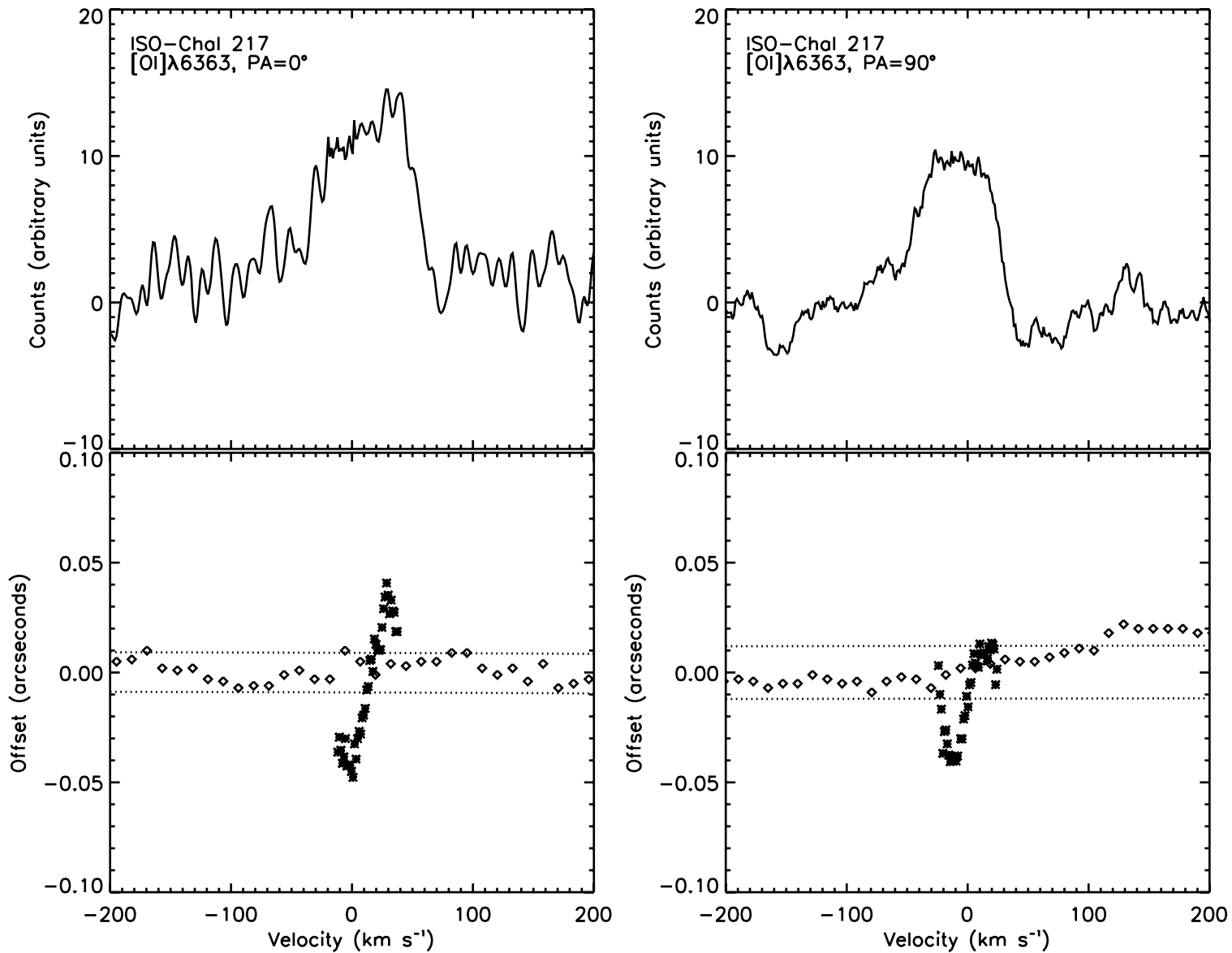

Figure 4. Spectro-astrometric analysis of the ISO-Cha217 [O I] 26363 line. Analogous to the [O I] $\lambda 6300$ line, the line is made of blue- and redshifted emission which spectro-astrometry shows to be offset in opposing directions. As expected, the offsets are similar to what is measured for the [O I] $\lambda 6300$ line. Again comparison between measurements at $0^{\circ}$ and $90^{\circ}$ constrain the outflow P.A. at $\sim 205^{\circ}$. As for all the ISO-ChaI $217 \mathrm{FELs}$, offsets are a moving sum across the continuum and line position and the $1 \sigma$ error in both measurements is comparable.

intensity. Where the spectro-astrometric technique measures the spatial mean of the feature to be, is influenced by where the maximum intensity feature lies. In other words, an unresolved jet will be measured to be less extended than it really is (using spectro-astrometry), if it actually consists of bright inner knots and lower brightness material further out. For the above reasons, the estimates of the P.A.s of the ISO-ChaI 217 and ISOOph 32 outflows given here are a first approximation and the errors quoted are taken from the spectro-astrometric uncertainty (dependent on $\mathrm{S} / \mathrm{N}$ ). Also note that outflow P.A.s measured here only apply to the outflow as studied close to its driving source. It is possible that some bending may occur in the outflow at large distances which will change the outflow P.A. (Fendt \& Zinnecker 1998). However, we expect any such variation to be within the errors quoted in Table 3. Below, the results for ISO-Cha1 217, ISO-Oph 32, and DENIS-P J160603.9-20564 are presented separately. Tables 2 and 3 give the FELs detected for each source along with the radial velocity (measured with respect to the systemic velocity), offset measured, and estimate of the outflow P.A.

\subsection{ISO-ChaI 217}

ISO-ChaI 217 was first reported as part of an ISOCAM survey of the Chamaeleon I dark cloud (Persi et al. 2000). Current estimates place its mass at $80 M_{\mathrm{JUP}}$ (Muzerolle et al. 2005) and its spectral type between M7.5 and M6.25 (López
Martí et al. 2004; Muzerolle et al. 2005). Hence, it currently lies on the boundary between being a BD or an ultra-low mass star. The accretion properties of ISO-ChaI 217 have been much studied in recent years. Apai et al. (2005) confirm midinfrared excess emission, indicative of the presence of a disk and Scholz \& Jayawardhana (2006) demonstrate that it is a strong accretor which exhibits significant variability in its accretion activity. Scholz \& Jayawardhana (2006) also observe signatures of outflow activity in their spectra of ISO-Cha1 217. These signatures are in the form of FELs of S. Here, the origin of these (and other) FELs in an outflow driven by ISO-ChaI 217 is confirmed for the first time.

UVES spectra of ISO-ChaI 217, taken at orthogonal slit positions were obtained on May 1 2007. Strong [S II] $\lambda \lambda 6716,6731$, [O I $] \lambda \lambda 6300,6363$, and $\mathrm{H} \alpha$ emission was detected at both P.A.s along with weak $\left[\mathrm{N}_{\mathrm{II}}\right] \lambda 6548$ emission. Blue- and redshifted components to the $\left[\mathrm{S}\right.$ II] $\lambda \lambda 6716,6731$ and $\left[\mathrm{O}_{\mathrm{I}}\right] \lambda \lambda 6300,6363$ lines were detected at an average radial velocity (with respect to the systemic velocity) of -16 and $32 \mathrm{~km} \mathrm{~s}^{-1}$. The systemic velocity of ISO-ChaI 217 is taken as $+2 \mathrm{~km} \mathrm{~s}^{-1}$ (James et al. 2006; Bary et al. 2008). The [S II] $\lambda \lambda 6716,6731$, [O I] $\lambda \lambda 6300,6363$, and $\mathrm{H} \alpha$ lines were analyzed with spectro-astrometry and the analysis revealed the blue- and redshifted emission to be offset in opposing directions. Hence, ISO-Cha I 217 is driving a bipolar outflow (see Figures 3-7). As is the case in CTTS, the [S II] $\lambda \lambda 6716,6731$ lines trace the outflow further from the source than the $\left[\mathrm{O}_{\mathrm{I}}\right] \lambda \lambda 6300,6363$ lines (refer to Table 2). Emission is 

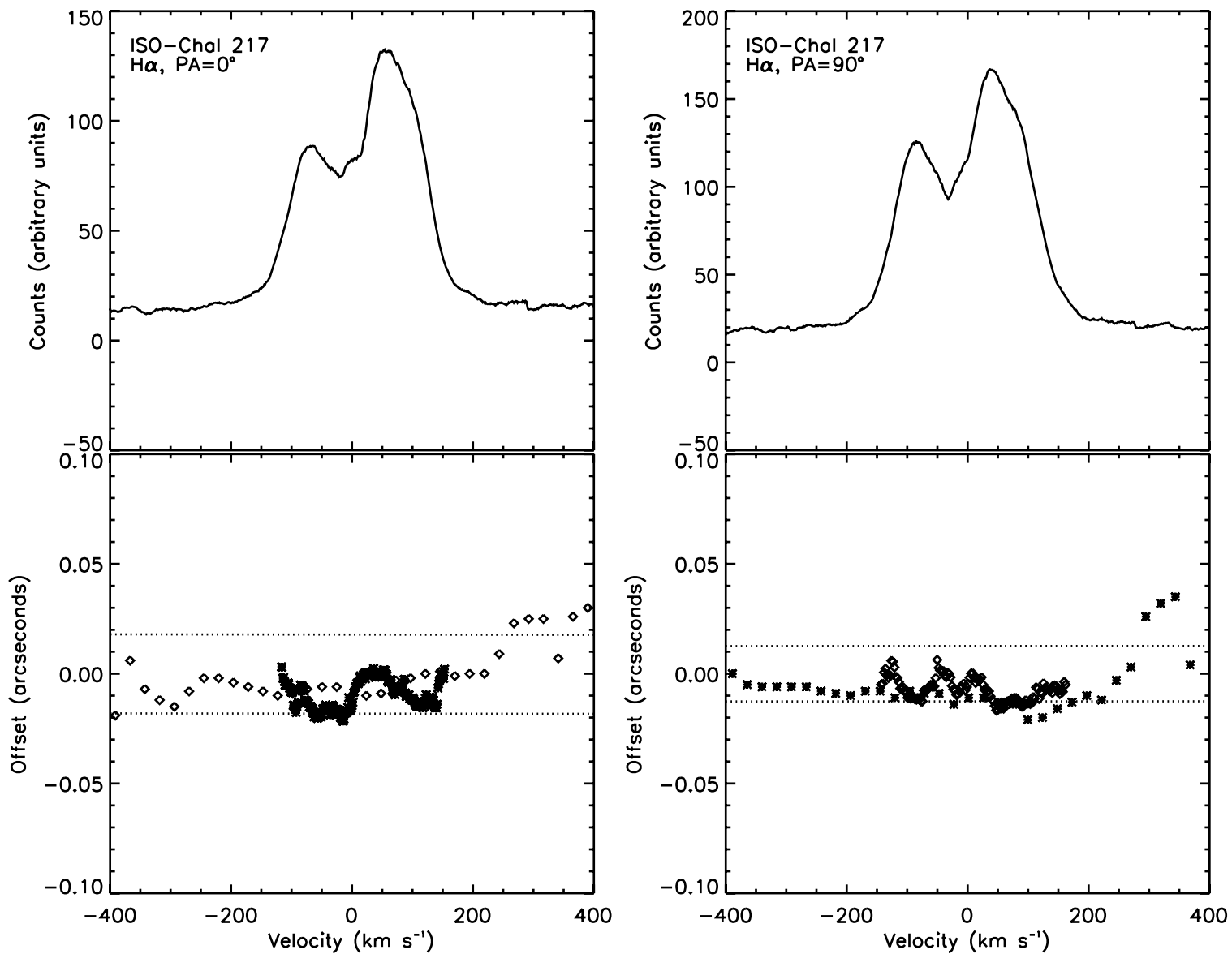

Figure 5. Spectro-astrometric analysis of the ISO-Cha1 $217 \mathrm{H} \alpha$ line. Analysis was carried out in the same manner, as outlined for the [OI] $\lambda \lambda 6300,6363$ lines. No signal from the outflow is detected in the $\mathrm{H} \alpha$ line. Emission is coincident with the source hence it is reasonable to assume that the bulk of the emission is coming from the accretion flow. For the $\mathrm{H} \alpha$ line, the spectro-astrometric accuracy achieved at the line peak is much greater than in the wings or for the continuum emission. Hence, for all the $\mathrm{H} \alpha$ lines emission is summed so that the error in the line-wing and continuum is comparable.

clearly more extended at $0^{\circ}$ than at $90^{\circ}$ and a comparison between the two is used to constrain the outflow P.A. to a range of $193^{\circ}-209^{\circ}$ (E of N). Note that the lines which trace the outflow closest to the source give a slightly larger P.A. on average than the more displaced line regions (Table 3), although all values agree within the errors. Analysis of the $\mathrm{H} \alpha$ line reveals no detectable contribution from the outflow. Clearly, the $\mathrm{H} \alpha$ line is coincident with the continuum position at both P.A.s.

A significant feature is the obvious asymmetry in the outflow as traced by $[\mathrm{S} I \mathrm{II}]$. The redshifted lobe is much brighter in $\left[S_{\text {II }}\right] \lambda \lambda 6716,6731$ than the blueshifted and there is a velocity difference of a factor of 2 between the red and the blue lobe. This is especially apparent in the case of the brighter [S II] 66731 line at a P.A. of $0^{\circ}$ (Figure 7). Although velocity and intensity asymmetries are commonly seen in CTT jets (Hirth et al. 1994) and while several case have been documented where the redshifted lobe is the faster, it is unusual for the redshifted lobe to be the brightest. In the majority of CTT jets, the circumstellar disk obscures the redshifted flow. The CTTS RW Aur offers the most striking example of such an asymmetry (Hirth et al. 1994; López-Martín et al. 2003). In this case, a similar velocity asymmetry has been reported but the slower lobe (red) is the brightest. The ISO-ChaI 217 asymmetry is clearly visible in the PV diagrams presented in Figure 8. For the purpose of the PV diagrams, the $\left[\mathrm{S}_{\mathrm{II}}\right] \lambda 6731$ line region was smoothed, using an elliptical Gaussian filter. The PV diagrams support the spectro-astrometric analysis as the opposing offset in the line region at $0^{\circ}$, measured with spectro-astrometry to be $\sim 180$ mas, is resolved in the corresponding PV plot. In the PV plot at $90^{\circ}$, the line appears coincident with the source position again consistent with the much smaller offsets measured at this slit position ( $\sim 50$ mas).

\subsection{ISO-Oph 32}

Natta et al. (2002) observed this source in the near-infrared and estimated its spectral type and mass at M7.5 and 30$50 M_{\mathrm{JUP}}$. They also argue that the mid-infrared excess detected in this object is consistent with the presence of an irradiated disk. In a later study, Natta et al. (2004) added optical spectra to their near-infrared data. In particular, they use the $\mathrm{H} \alpha$ line as a test of accretion activity. In the both the $0^{\circ}$ and $90^{\circ}$, high-resolution UVES spectrum presented here the $[\mathrm{O} \mathrm{I}] \lambda 6300$ line is detected at at $\mathrm{S} / \mathrm{N}$ of just above 4 . As the [O I] 26363 line is 3.5 times fainter than the [O I] 26300 line, it is marginally detected at both PAs and therefore not analyzed with spectro-astrometry. The lower critical density lines are below the detection limit in our spectra. As shown in Figure 9, analysis reveals the [O I] 26300 line to be offset in a southerly direction to $\sim 60$ mas at $0^{\circ}$ and $\sim 105$ mas at $90^{\circ}$. The average radial velocity (with respect to the systemic velocity) of the two lines is $15 \mathrm{~km} \mathrm{~s}^{-1}$ and comparison of the measured offsets at the orthogonal slit positions constrains the P.A. of the blueshifted outflow at $\sim 240^{\circ}$. The systemic velocity of ISO-Oph 32 is taken as $+7 \mathrm{~km} \mathrm{~s}^{-1}$ by Whelan et al. (2005). 

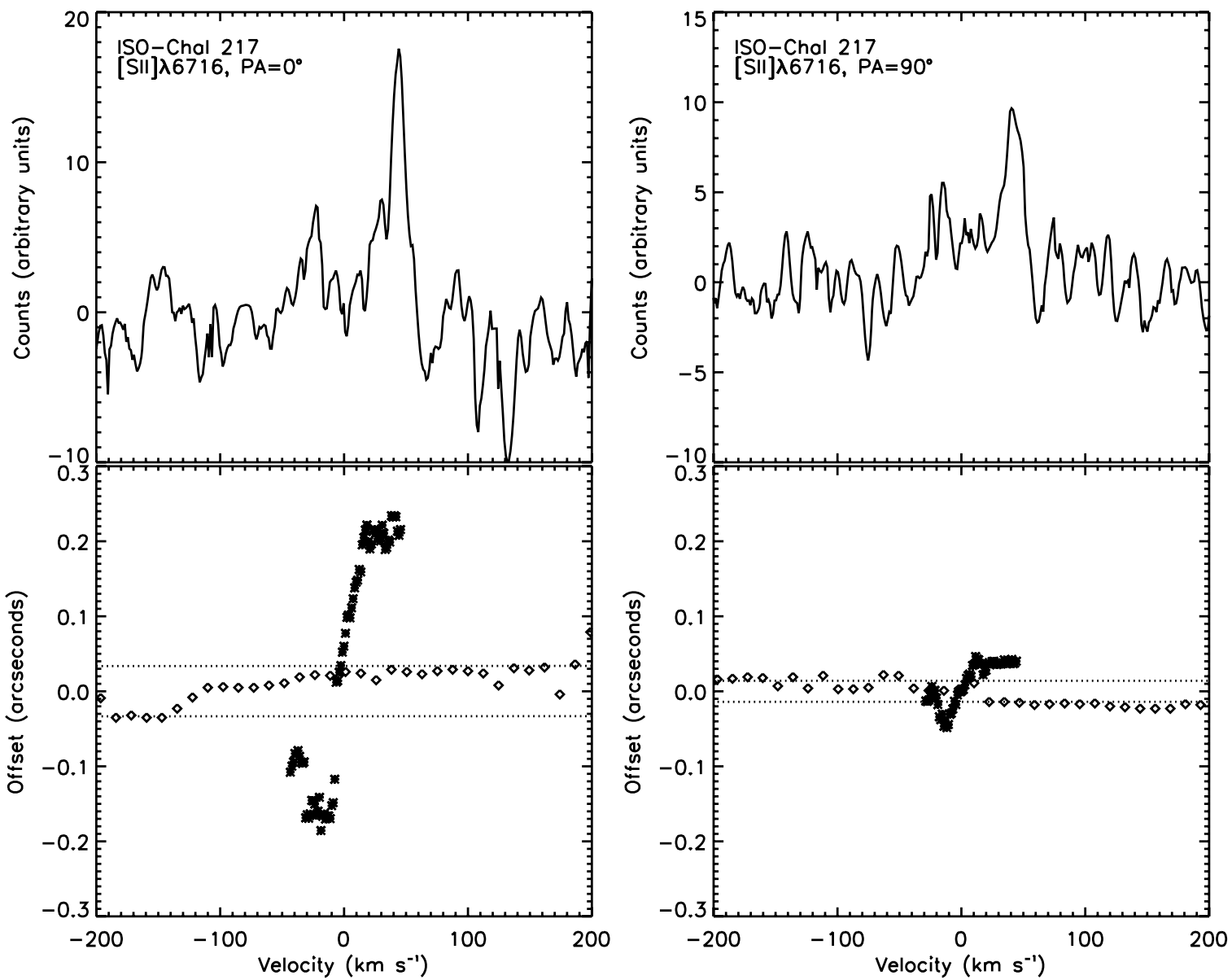

Figure 6. Spectro-astrometric analysis of the ISO-Cha1 $217[\mathrm{~S} \mathrm{II}] \lambda 6716$ line. Again the line was observed at a P.A. of $0^{\circ}$ and $90^{\circ}$. The blue- and redshifted emission is more clearly separated spectrally than in the [O I] $\lambda \lambda 6300,6363$ lines. In addition, it is offset further from the BD position. Analysis of the [S II] $\lambda 6716$ line constrains the outflow P.A. at $\sim 193^{\circ}$. This is smaller than but similar to the estimate from the [OI] $\lambda \lambda 6300,6363$ lines. Note the asymmetry in the red and blue lobes of the outflow in both velocity and intensity.

Again no spectro-astrometric signal is detected in the $\mathrm{H} \alpha$ line, confirming the origin of the bulk of the emission in the accretion flow (see Figure 10).

\subsection{DENIS-P J160603.9-205644}

DENIS-P J160603.9-205644 was identified as a BD as part of a survey of the Upper Scorpius OB association Martín et al. (2004). Mohanty et al. (2005) classify it as an accretor ( $\mathrm{H} \alpha 10 \%$ width is given as $306 \mathrm{~km} \mathrm{~s}^{-1}$ ) and give its spectral type and mass as M7.5 and $40 M_{\mathrm{JUP}}$. However, no FELs or additional signatures of outflow activity are found in the spectra of DENIS-P J160603.9-205644. The spectro-astrometric analysis of the $\mathrm{H} \alpha$ line at $0^{\circ}$ and $90^{\circ}$ is shown in Figure 11. While the line profile is asymmetric the emission is coincident with the continuum and therefore must originate at the star. The lack of a spectro-astrometric signal in the $\mathrm{H} \alpha$ line rules out any complications with spectro-astrometric artifacts. Although DENIS-P J160603.9-205644 is classified as an accretor, it is likely that it is the least accreting object in our sample. Mohanty et al. (2005) give upper limits to its mass accretion rate based on the non detection of the Ca II $8662 \AA$ feature. Herczeg et al. (2009) measure $\dot{M}_{\text {acc }}$ for this object at $2.5 \times 10^{-12} M_{\odot} \mathrm{yr}^{-1}$ consistent with the upper limits set by Mohanty et al. (2005). Also note that Herczeg et al. (2009) do detect some faint forbidden emission in the spectrum of DENIS-P J160603.9205644. Their observations were made with LIRIS on Keck I at a spectral resolution of $R=1400$. Given the relatively high spectral resolution of our observations and the small accretion rate of this object, it is not surprising that no detection is made here.

\section{MEASURING THE MASS OUTFLOW RATE FOR BD OUTFLOWS}

Numerous observational studies have constrained the mass outflow rate $\left(\dot{M}_{\text {out }}\right)$ in CTTSs at $\sim 1-10 \%$ of the mass accretion rate (Hartigan et al. 1994; Podio et al. 2006; Garcia Lopez et al. 2008). The ratio between the mass outflow and accretion rates $\left(M_{\text {out }} / M_{\text {acc }}\right)$ in young stars is a defining characteristic of the mechanism which is at work to launch protostellar outflows (Ferreira et al. 2006; Ray et al. 2007). If a similar ratio between outflow and infall is measured for accreting BDs it will provide compelling evidence that the outflows we are beginning to detect at very low and sub-stellar masses are in fact scaled-down from low-mass protostellar outflows. The spectra of low-mass protostars contain a wealth of forbidden and permitted lines whose excitation properties are well known. Hence, the ratios and intensities of these lines can be used to probe the physical conditions of the emitting regions. Spectral analysis of known outflow tracers, typically in the optical and near-infrared regimes, has allowed $M_{\text {out }}$ to be estimated for low-mass protostars. In comparison, the spectra of young BDs, have been little investigated. It is only in the last number of years that optical FELs have been detected in 

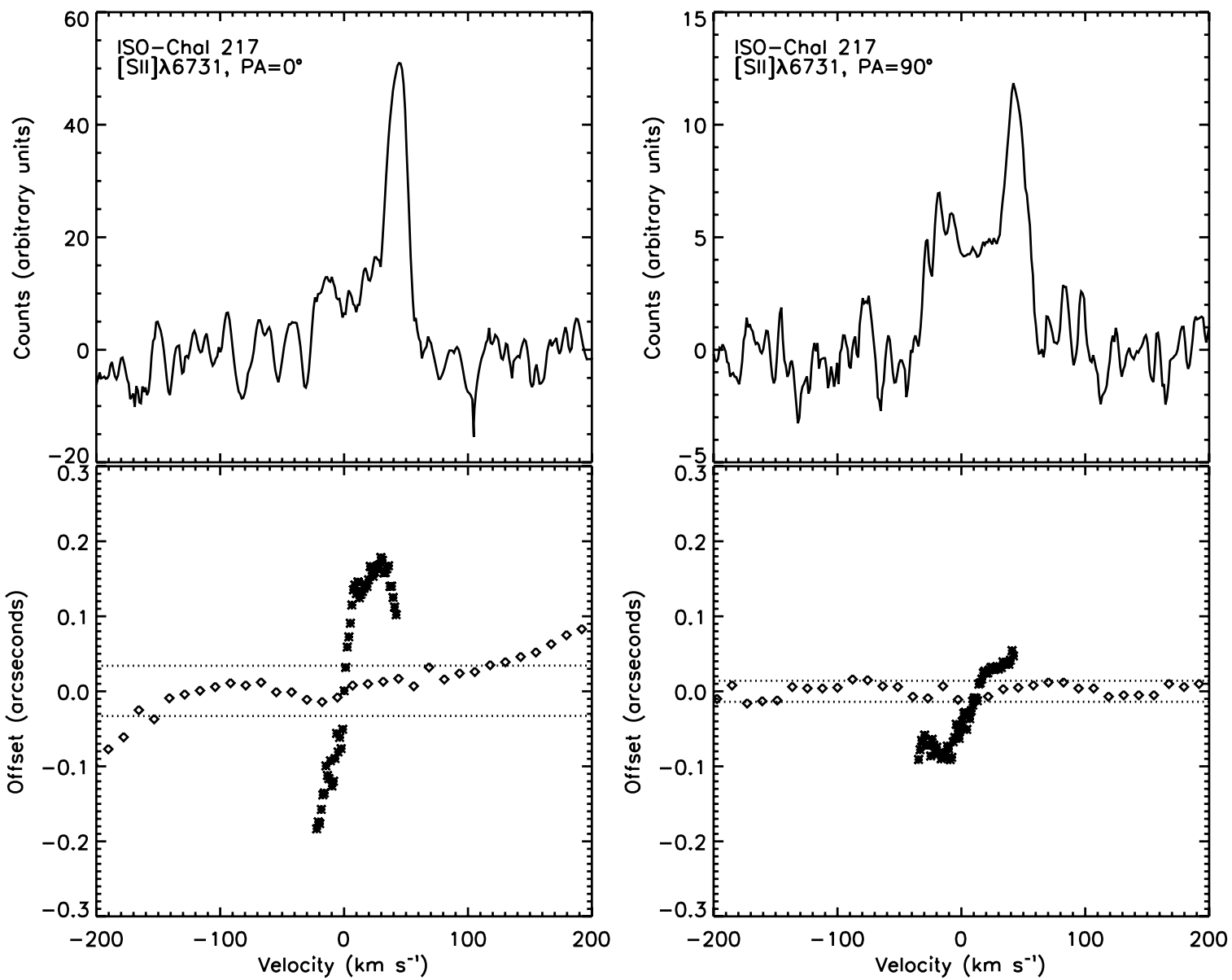

Figure 7. Spectro-astrometric analysis of the ISO-Cha1 217 [S II] 26731 line. Analysis agrees with results for the [S II] $\lambda 6716$ line and constrains the outflow P.A. at $\sim 200^{\circ}$. Again the red and blue lobes of the outflow are clearly separated and the asymmetry is especially clear. The difference between the intensity of the red and blue lobes as traced by the $[\mathrm{S} \mathrm{II}] \lambda 6731$ line at $0^{\circ}$ is striking.

BD spectra and comparatively little work has been done in the near-infrared.

Two distinct methods have been used to measure $\dot{M}_{\text {out }}$ in low-mass protostars. Here, the application of these methods to the problem of measuring mass outflow rates at $\mathrm{BD}$ masses, is discussed. The first method (hereafter method A) relies on an estimate of the total density in the outflow $n_{H}$, which in turns requires the electron density $\left(n_{e}\right)$ and ionization fraction $\left(x_{e}\right)$ to be known (Nisini et al. 2005; Podio et al. 2006). These plasma parameters can be calculated using the Bacciotti \& Eislöffel (BE) technique (Bacciotti \& Eislöffel 1999; Bacciotti et al. 1999). The BE technique employs selected optical transitions of $\mathrm{S}^{+}, \mathrm{O}^{0}$, and $\mathrm{N}^{+}$, to derive $x_{e}$ and $T_{e}$ (electron temperature). The electron density is estimated from the ratio of the [S II] lines, [S II] $\lambda 6716 /\left[\mathrm{S}_{\text {II }}\right] \lambda 6731$ (Osterbrock 1994) and once this quantity is known it is combined with $x_{e}$ to derive $n_{H}$. Podio et al. (2006) adopt the BE technique to study the physical conditions in a sample of protostellar outflows and thus calculate their mass loss rate. As the $\mathrm{BE}$ technique calculates $T_{e}$ and $x_{e}$ from the $[\mathrm{N} \mathrm{II}] \lambda \lambda(6548+6583) /[\mathrm{O} \mathrm{I}] \lambda \lambda(6300+6363)$ and $[\mathrm{S} \mathrm{II}] \lambda \lambda(6716+6731) /[\mathrm{O} \mathrm{I}] \lambda \lambda(6300+6363)$ line ratios, it requires the use of a given set of elemental abundances. Podio et al. (2006) investigate any variations in estimates of $x_{e}$ and $T_{e}$ for four different abundance sets and calculate $\dot{M}_{\text {out }}$ using,

$$
\dot{M}_{\text {out }}=\mu m_{H} n_{H} \pi r_{J}^{2} v_{J},
$$

where $\mu=1.41$ is the mean atomic weight, $m_{H}$ the mass of a proton, $n_{H}$ the total density and $r_{J}, v_{J}$ the jet radius and velocity, respectively. Here, we firstly discuss the analysis of our BD spectra with the BE technique.

The BE technique is easily applied to the spectrum of a typical CTTS where the required FELs are well resolved spatially and spectrally with a high $\mathrm{S} / \mathrm{N}$. The line flux can be measured using several different line fitting techniques. The main source of error comes from the uncertainty in the measurement of the line fluxes which depends on the $\mathrm{S} / \mathrm{N}$ of the line. In order to use the BE technique to investigate a BD outflow, the [O I] $\lambda 6300$, [S II] 66716,6731 and [N $\left.{ }_{\text {II }}\right] \lambda 6583$ lines must all be detected at a sufficient $\mathrm{S} / \mathrm{N}$. The $\left[\mathrm{O}_{\mathrm{I}}\right] \lambda 6363$ and $[\mathrm{N}$ II] $] 2548$ lines can be substituted for the $\left[\mathrm{O}_{\mathrm{I}}\right] \lambda 6300,\left[\mathrm{~N}_{\mathrm{II}}\right] \lambda 6583$ lines, if for example the brighter [OI] 6300 line is not covered by the spectral range of the instrument or the $\left[\mathrm{N}_{\mathrm{II}}\right] \lambda 6548$ is blended with the $\mathrm{H} \alpha$ line. Both the $\left[\mathrm{O}_{\mathrm{I}}\right] \lambda 6300$ and $\left[\mathrm{N}_{\mathrm{II}}\right] \lambda 6548$ lines can be exchanged with the fainter line of their pairs, as the ratio of their intensities is always three. This is due to the fact that they come from the same upper level and that the ratio of the A coefficients for spontaneous emission is three. Of the five BD candidates found so far to have outflows only one, LSRCrA 1 (outflow discussed in Whelan et al. 2009), meets the requirements of the BE technique. For the two lowest mass BDs, ISO-Oph 32 and 2MASS1207-3932 (Whelan et al. 2007) only the $[\mathrm{O}$ I] $\lambda \lambda 26300,6363$ lines are observed. An added difficulty for 2MASS1207-3932 is that while both the blue- and redshifted 
$[S I I] \lambda 67310^{\circ}$

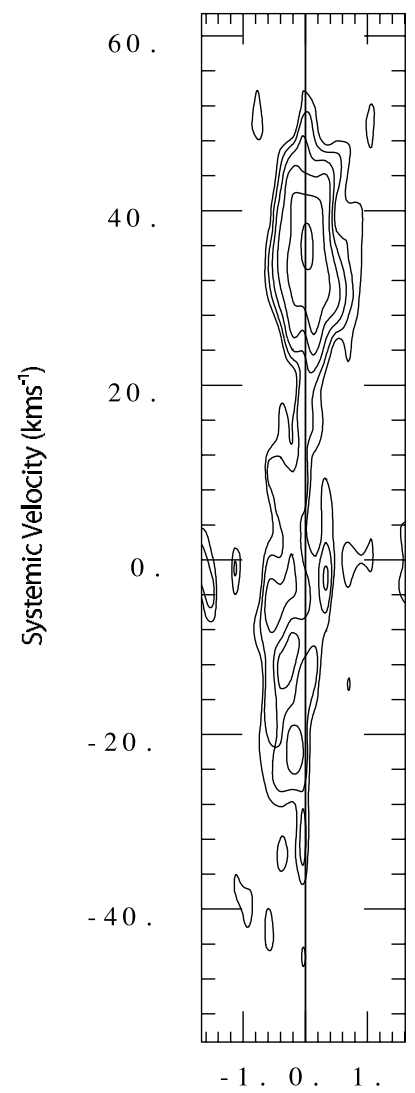

Offset (arcsec)
$[\mathrm{SII}] \lambda 673190^{\circ}$

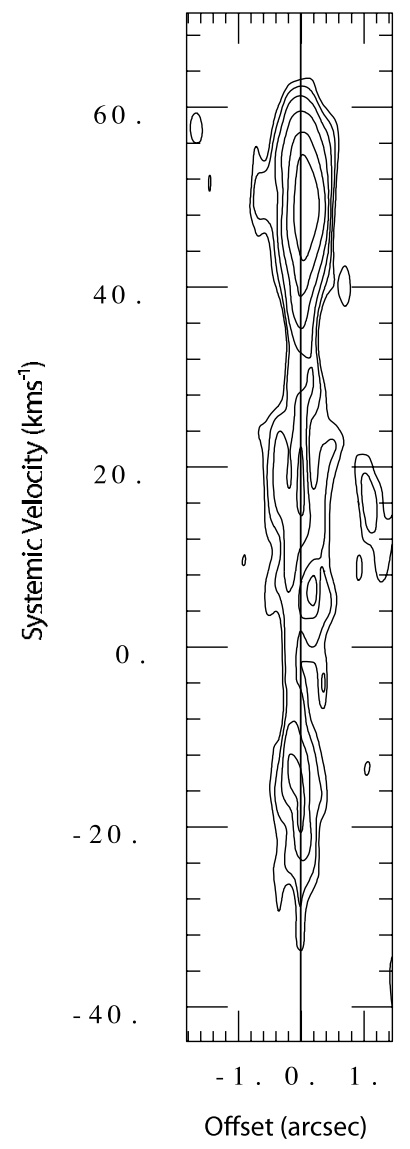

Figure 8. Position-velocity diagrams of the ISO-ChaI 217 [S II] ${ }^{2} 6731$ line region, comparing the line at slit P.A.s of $0^{\circ}$ (left) and at $90^{\circ}$ (right). For the PV diagrams, the emission line region was smoothed using an elliptical Gaussian filter as described above. Contours begin at 3 times the rms noise and increase by factors of $\sqrt{2}$. The black line delineates the BD position. The PV plots clearly support the spectro-astrometric results. The relative offset between the red- and blueshifted part of the line can be seen by eye in the plot for $0^{\circ}$. As this offset is a factor of 4 smaller at $90^{\circ}$, it is not obvious in the corresponding PV diagram. A difference in brightness between the redshifted components is also apparent with the redshifted component at $90^{\circ}$ appearing marginally brighter. As we are only detecting emission very close to ISO-Cha1 217 and given that our slit width is $1^{\prime \prime}$ we would not expect to see any significant variation in brightness with P.A. The apparent difference here is due to a change in observing conditions between the acquisition of the two spectra with the seeing decreasing from $0 . " 85$ for the $0^{\circ}$ spectrum to $0{ }^{\prime \prime} 7$ at $90^{\circ}$. We measure the integrated flux of the redshifted components to be comparable with the value being $0.2,0.45 \times 10^{-16} \mathrm{erg} \mathrm{s}^{-1}$ $\mathrm{cm}^{2} \mathrm{~A}^{-1}$ and $0.21,0.5 \times 10^{-16} \mathrm{erg} \mathrm{s}^{-1} \mathrm{~cm}^{2} \mathrm{~A}^{-1}$ for the red/blueshifted components at $0 / 90$ degrees, respectively.

lobes of the outflow are detected in the [O I] 66300 line they are not well resolved spectrally, hence the emission from both lobes cannot be properly separated. While both the [O I] $\lambda \lambda 26300,6363$ and $\left[\mathrm{S}_{\mathrm{II}}\right] \lambda 6731$ lines are found in the spectrum of ISO-Oph 102 (Whelan et al. 2005), the [S II] 26716 line is not detected and therefore there is no estimate of $n_{e}$. Finally, for ISO-ChaI 217 , while the $\left[\mathrm{O}_{\mathrm{I}}\right] \lambda 6300$ and $\left[\mathrm{S}_{\mathrm{II}}\right] \lambda \lambda 6716,6731$ lines are all observed the $\left[\mathrm{N}_{\text {III }}\right] \lambda 6548$ line is not present and the $\left[\mathrm{N}_{\text {II }}\right] \lambda 6583$ line is too faint to allow the use of the BE technique. However, using the ratio of the [S II] $] \lambda \lambda 6716,6731$ lines $n_{e}$ in the blue- and redshifted lobes of the ISO-ChaI 217 outflow is measured (see Table 4).

The results of the application of the $\mathrm{BE}$ technique to the blueshifted outflow driven by LS-RCrA 1 are reported in Table 4. As described in Whelan et al. (2009), UVES spectra of LS-RCrA 1 were taken at several P.A.s between $45^{\circ}$ and $82^{\circ}$ and analysis of this data constrained the P.A. of the LS-RCrA 1 outflow at $\sim 15^{\circ}$. Using the $\mathrm{BE}$ technique, $n_{e}, x_{e}, T_{e}$, and $n_{H}$ are derived at each P.A. The quoted errors come from the uncertainty in the measurement of the line fluxes. In the case of low-mass protostars, values of $x_{e}$ in the range $0.01-0.4, T_{e}$ in the range $8000-20,000 \mathrm{~K}$ and $n_{H}$ up to $10^{6} \mathrm{~cm}^{-3}$ are typically measured (Ray et al. 2007). The estimates of $n_{e}$ for LS-RCrA 1 and ISOChal 217 are reasonable when compared with estimates made for CTT jets and the large errors (up to $\sim 40 \%$ ) are due to the low $\mathrm{S} / \mathrm{N}$ in the $[\mathrm{S} \mathrm{II}]$ lines, which is a low as 3 in some cases. Comerón et al. (2003) quoted a value of $n_{e}=2300 \mathrm{~cm}^{-3}$ for LS-RCrA 1. Note that the ISO-ChaI 217 redshifted outflow has a higher electron density than the blueshifted, which is a further indication of the asymmetry between the two lobes of the outflow. The low S/N of the LS-RCrA 1 [N II] 26583 line leads to errors of up to $50 \%$ in the derived values of $x_{e}$ and $n_{H}$. The estimates of $x_{e}$ are very low compared to what one measures for CTTSs and decrease as the slit P.A. moves away from the outflow P.A. In addition, values of $T_{e}$ and $n_{H}$ are very high. With such high densities, one would expect more efficient cooling and therefore lower temperatures. The peculiarities in $x_{e}, T_{e}$, and $n_{H}$ noted here have been observed in CTT flows where the very beginning of the flow $\left(0^{\prime \prime} .2\right.$ to $\left.1^{\prime \prime}\right)$ is being tested with $\mathrm{BE}$ technique. In the case of the BDs, we are probing even closer to the driving source of the outflow. Bacciotti et al. (1999) argue that the physical reason for this behavior could be related to the event that creates the ionization of the flow, e.g., a standing shock. While these preliminary results are interesting, it is impossible at present to say if this approach can reveal anything meaningful about the ionization fraction/electron temperature and thus shocked emission in $\mathrm{BD}$ outflows. Higher S/N spectra of a bigger sample of young BD outflows, aimed at resolving the $[\mathrm{N}$ II] lines in particular, should be the next step.

The value of $n_{H}$ estimated from the above method is used in Equation (1) to derive $\dot{M}_{\text {out }}$. As the slit P.A. of $45^{\circ}$ lies closest to the estimated outflow P.A. $\left(15^{\circ}\right), n_{H}$ derived for this slit P.A. is taken. One of the biggest difficulties with measuring $M_{\text {out }}$ for $\mathrm{BD}$ outflows is that the outflow width and velocity is not known. Here, reasonable estimates of $r_{J}, v_{J}$ for LS-RCrA 1 are made. Ray et al. (2007) show a plot of jet width as a function of projected distance from the source. Assuming a similar relationship for a young $\mathrm{BD}$ outflow and considering that offsets of up to 150 mas ( $20 \mathrm{AU})$ have been measured for the LS-RCrA 1 FELs, $10 \mathrm{AU}$ is taken as a reasoned approximation of the radius of the LS-RCrA 1 outflow. This would suggest an opening angle close to $30^{\circ}$ for this outflow and is likely an upper limit on the outflow radius. The radial velocities of the LS-RCrA 1 FELs are low $\left(\sim 25 \mathrm{~km} \mathrm{~s}^{-1}\right)$ and at present the inclination angle of the outflow is poorly constrained. Initial observations suggested that the low radial velocity of the FELs and the faintness of the source could be explained by an edge-on accretion disk and thus an outflow inclined close to the plane of the sky. However, the lack of redshifted emission and the evidence for separation between low and high velocity emission in the outflow, presented in Whelan et al. (2009), refute this argument. Assuming that the low radial velocity measured in the outflow tracers suggests a low outflow inclination angle $\left(20^{\circ}-30^{\circ}\right)$, for the purpose of calculating $\dot{M}_{\text {out }}, v_{J}$ is taken at $75 \mathrm{~km} \mathrm{~s}^{-1}$. Combining $n_{H}$ with $r_{J}$ and $v_{J}$ yields $\dot{M}_{\text {out }}=2.4 \times$ $10^{-9} M_{\odot} \mathrm{yr}^{-1}$.

The second widely used method for measuring $\dot{M}_{\text {out }}$ (hereafter method B) is based on the observed luminosity $L$ (line) of an 

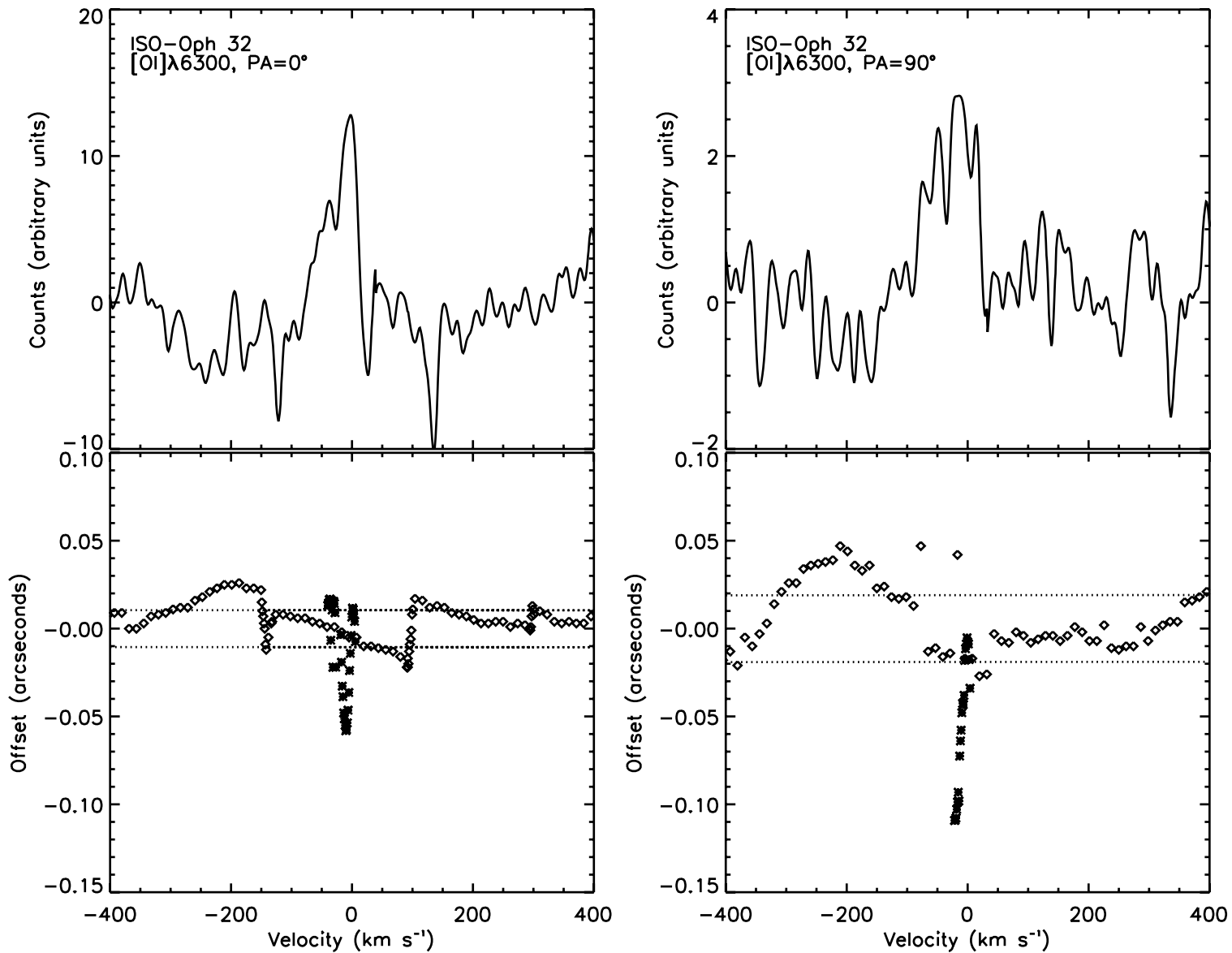

Figure 9. [OI] 6300 line emission and related spectro-astrometric analysis for the young BD ISO-Oph 32. As reported for other BDs, the [OI] 6300 line was the only FEL found in the spectrum strong enough for spectro-astrometric analysis. Spectro-astrometry confirms the origin of the line in an outflow. The measured offset in the line is greater at $90^{\circ}$ than at $0^{\circ}$ and from this comparison the outflow P.A. is estimated at $240^{\circ}$. In the case of ISO-Oph 32 , the line and continuum regions were smoothed rather than summed.

optically thin line such as [S II] or [O I]. The luminosity of a specific line can be derived as follows (Comerón et al. 2003)

$$
L\left(L_{\odot}\right)=6.71 \times 10^{-5} D^{2}(p c) \mathrm{EW}(\AA) 10^{-0.4 R_{0}}
$$

( $D$ is the distance to the object, EW the equivalent width of the line, and $R_{0}$ the dereddened magnitude) and used to evaluate the mass of the flow $M$ (in the aperture) according to the following equations (Hartigan et al. 1995),

$$
\begin{aligned}
& M=9.61 \times 10^{-6}\left(1+\frac{n_{c}}{n_{e}}\right)\left(\frac{L_{6300}}{L_{\odot}}\right) M_{\odot}, \\
& M=1.43 \times 10^{-3}\left(1+\frac{n_{c}}{n_{e}}\right)\left(\frac{L_{6731}}{L_{\odot}}\right) M_{\odot} .
\end{aligned}
$$

$\dot{M}_{\text {out }}=M V_{\tan } / 1_{\tan }$, where $n_{c}, V_{\tan }$, and $l_{\tan }$ are the critical density, outflow tangential velocity, and the size of the aperture in the plane of the sky. As $n_{e}$ for CTTSs is comparable to the critical density of [S II] $\lambda 6731$, Hartigan et al. (1995) remove the critical density dependence from Equation (4) above. For the BD outflows investigated to date $n_{e}<n_{c}$, hence this dependence is included here.

In order to measure $L$ (line) and apply the above method to our sources, $R_{0}, n_{e}$, and $V_{\tan }$ must be known. Here, we estimate $\dot{M}_{\text {out }}$ for LS-RCrA 1, ISO-ChaI 217, and ISO-Oph 102. The values of EW, $R_{0}, n_{e}$, and $V_{\tan }$ used in each case are given in Table 5.
For LS-RCrA $1, \dot{M}_{\text {out }}$ is measured at $6.1 \times 10^{-10} M_{\odot} \mathrm{yr}^{-1}$ and $2.0 \times 10^{-10} M_{\odot} \mathrm{yr}^{-1}$ from the [O I] $\lambda 6300$ and [S II] 26731 lines, respectively. $V_{\tan }$ is taken at $-43 \mathrm{~km} \mathrm{~s}^{-1}$. This estimate is based on a radial velocity of $25 \mathrm{~km} \mathrm{~s}^{-1}$ and an outflow inclination angle of $30^{\circ}$ (see above). For the ISO-ChaI 217 bipolar outflow we chose to estimate $\dot{M}_{\text {out }}$ from the [S II] $\lambda 6731$ line luminosity. As the outflow is resolved spectrally in the $[\mathrm{S}$ II $] \lambda 6731$ line (the lobes are blended spectrally in the [O I] 26300 line), it was possible to estimate $\dot{M}_{\text {out }}$ in both lobes of the outflow. For the faster (redshifted lobe) $\dot{M}_{\text {out }}=3.1 \times 10^{-10} M_{\odot} \mathrm{yr}^{-1}$. This is almost twice the outflow rate of the blue lobe, which is measured at $1.8 \times 10^{-10} M_{\odot} \mathrm{yr}^{-1}$. A similar difference in $\dot{M}_{\text {out }}$ is observed in the two lobes of the RW Aur asymmetric jet Woitas et al. (2002). $R_{0}$ for ISO-ChaI 217 is taken from the $R=19.59 \mathrm{mag}$ (López Martí et al. 2004) and $A_{J}$ given by Luhman (2004) as 0.7 mag. As we see, the redshifted component of the ISO-ChaI 217 outflow the outflow likely has a low inclination angle. Radial velocities of $\sim-20 \mathrm{~km} \mathrm{~s}^{-1}$ and $40 \mathrm{~km} \mathrm{~s}^{-1}$ are measured for the red and blue lobes, therefore tangential velocities of $-55 \mathrm{~km} \mathrm{~s}^{-1}$ and $110 \mathrm{~km} \mathrm{~s}^{-1}$ are assumed for the purpose of this calculation. This is based on an outflow inclination angle of $20^{\circ}$.

Finally, we also apply the method of Hartigan et al. (1995) to the blueshifted outflow driven by ISO-Oph 102. The [S II] 26731 line is detected at a low $\mathrm{S} / \mathrm{N}$ and its luminosity is estimated at $3.1 \times 10^{-7} L_{\odot}$. As the [S II] $\lambda 6716$ line is not detected, there is no estimate of $n_{e}$ for the outflow. However, as $n_{c}$ for the [S II] 6731 line is closer to the value of $n_{e}$ measured for CTT/BD outflows 

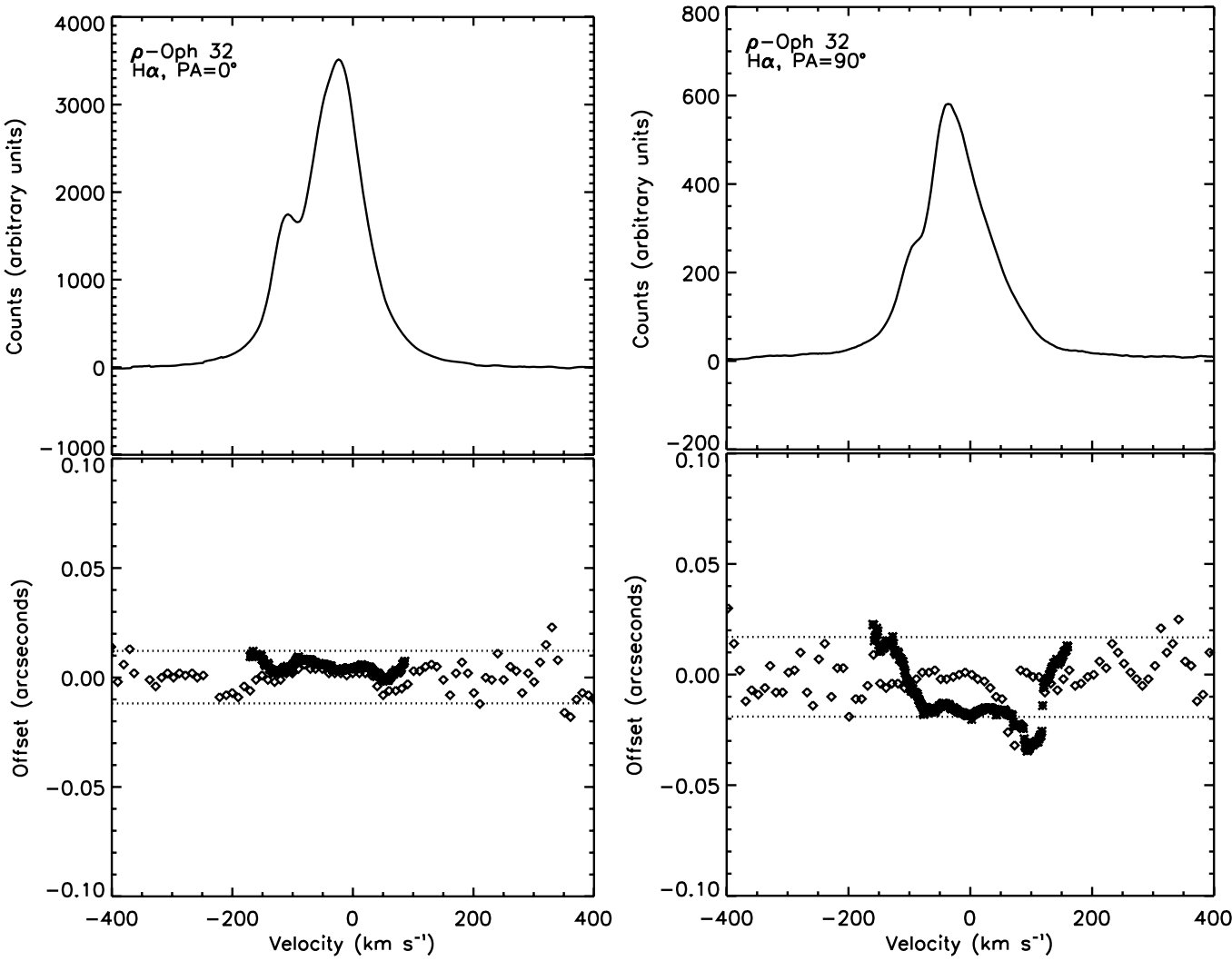

Figure 10. Spectro-astrometry of the ISO-Oph $32 \mathrm{H} \alpha$ line. As in the case of ISO-ChaI 217, offsets are a moving sum across the line and continuum position and the line and continuum are summed in such as way that the error in the line-wings and continuum is comparable. The $\pm 1 \sigma$ error is delineated by the dashed lines. As for all the sources discussed in this paper no signature of outflow activity is detected in the $\mathrm{H} \alpha$ line.
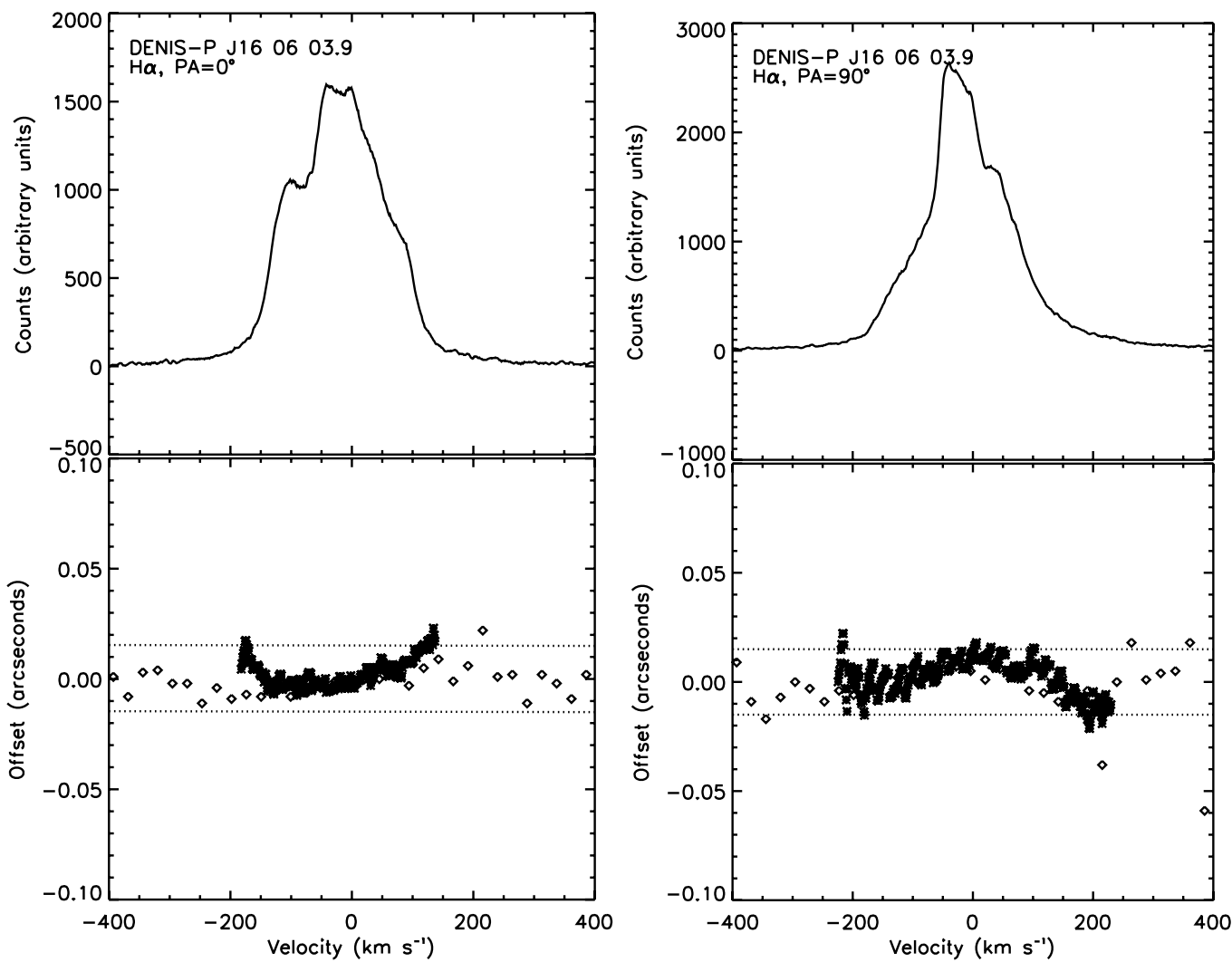

Figure 11. $\mathrm{H} \alpha$ line and spectro-astrometric analysis for DENIS-P J160603.9-205644. DENIS-P J160603.9-205644 is known to be actively accreting, however no evidence of outflow activity is found in our spectra. As is clear from the analysis shown here the $\mathrm{H} \alpha$ emission is coincident with the source position and therefore must originate very close to the source. This analysis was carried out in the same manner as for all the other $\mathrm{H} \alpha$ lines. 
Table 2

FELs of ISO-ChaI 217 and ISO-Oph 32 Detected at Slit P.A.s of $0^{\circ}$ and $90^{\circ}$

\begin{tabular}{|c|c|c|c|c|}
\hline Object & Slit P.A. $\left({ }^{\circ}\right)$ & Line & $V\left(\mathrm{~km} \mathrm{~s}^{-1}\right)$ & Offset (mas) \\
\hline \multirow{8}{*}{ ISO-ChaI 217} & 0 & {$[\mathrm{O}$ I $] \lambda 6300$} & $-20 / 30$ & $-50 / 50( \pm 6)$ \\
\hline & & {$[\mathrm{O}$ I $] \lambda 6363$} & $-10 / 28$ & $-50 / 45( \pm 9)$ \\
\hline & & {$\left[\mathrm{S}_{\text {II }}\right] \lambda 6716$} & $-20 / 42$ & $-180 / 200( \pm 33.5)$ \\
\hline & & {$[\mathrm{S}$ II $] \lambda 6731$} & $-20 / 38$ & $-180 / 180( \pm 33.5)$ \\
\hline & 90 & {$[\mathrm{O}$ I $] \lambda 6300$} & $-10 / 20$ & $-30 / 25( \pm 6)$ \\
\hline & & {$[\mathrm{O}$ I $] \lambda 6363$} & $-10 / 20$ & $-40 / 10( \pm 12)$ \\
\hline & & {$\left[\mathrm{S}_{\mathrm{II}}\right] \lambda 6716$} & $-15 / 40$ & $-46 / 40( \pm 14)$ \\
\hline & & {$\left[\mathrm{S}_{\mathrm{II}}\right] \lambda 6731$} & $-20 / 45$ & $-75 / 55( \pm 14)$ \\
\hline \multirow[t]{2}{*}{ ISO-Oph 32} & 0 & {$[\mathrm{O}$ I $] \lambda 6300$} & -10 & $-60( \pm 10)$ \\
\hline & 90 & {$\left[\mathrm{O}_{\mathrm{I}}\right] \lambda 6300$} & -20 & $-105( \pm 19)$ \\
\hline
\end{tabular}

Notes. Also given are the radial velocity of the lines (blue- and redshifted components in the case of ISO-ChaI 217) and the offsets in milliarcseconds recovered using spectro-astrometry. An estimate of the outflow P.A. is made by comparing the offsets measured at the orthogonal P.A.s.

(than for the $\left[\mathrm{O}_{\mathrm{I}}\right] \lambda 6300$ line), Equation (4) is less sensitive to the $n_{c} / n_{e}$ dependence and we can make a reasonable estimate of the possible range of $n_{e}$ for the ISO-Oph 102 outflow. Using a range of $10^{3} \mathrm{~cm}^{-3}$ to $10^{4} \mathrm{~cm}^{-3}$ for $n_{e}$ gives $\dot{M}_{\text {out }}$ at $(1.7-11.8)$ $\times 10^{-10} M_{\odot} \mathrm{yr}^{-1}$. A value of $V_{\tan }=78 \mathrm{~km} \mathrm{~s}^{-1}$ is assumed for this outflow based on the measured radial velocity of its FELs (Whelan et al. 2005) and on an outflow inclination of $30^{\circ}$. Phan-Bao et al. (2008) estimate the inclination angle of the
Table 3

Position Angles of the Two Outflows Observed

\begin{tabular}{ccc}
\hline \hline Object & Line & Outflow P.A. $\left(^{\circ}\right)$ \\
\hline ISO-ChaI 217 & {$[\mathrm{O} \mathrm{I}] \lambda 6300$} & $209( \pm 8)$ \\
& {$[\mathrm{O} \mathrm{I}] \lambda 6363$} & $206( \pm 15)$ \\
& {$[\mathrm{S} \mathrm{II}] \lambda 6716$} & $193( \pm 5)$ \\
& {$[\mathrm{S}$ II $] \lambda 6731$} & $200( \pm 6)$ \\
\hline ISO-Oph 32 & {$[\mathrm{O} \mathrm{I}] \lambda 6300$} & $240( \pm 7)$ \\
\hline
\end{tabular}

Notes. The P.A.s are estimated from orthogonal spectra as described in Section 3. For ISO-ChaI 217, a bipolar outflow is uncovered and estimates given above are an average of measurements made for the red- and blueshifted lobes. For ISO-Oph 32, only the blueshifted outflow is detected.

ISO-Oph 102 disk at $63^{\circ}$ to $66^{\circ}$. This suggests that the outflow inclination lies close to $30^{\circ}$ as assumed. $R_{0}$ is taken from Natta et al. (2004), where $A_{v}$ is estimated at $3 \mathrm{mag}$. Note that PhanBao et al. (2008) measure $\dot{M}_{\text {out }}=1.4 \times 10^{-9} M_{\odot} \mathrm{yr}^{-1}$ for the ISO-Oph 102 molecular outflow. It is reasonable that $\dot{M}_{\text {out }}$ for a molecular outflow be greater than the outflow rate in the underlying jet. Assuming that the jet is powering the molecular outflow (Downes \& Cabrit 2007), the mass outflow rate of the molecular component will grow with time as the jet transfers increasing amounts of energy and momentum.

Table 6 compares $\dot{M}_{\text {out }}$ and $\dot{M}_{\text {acc }}$ for LS-RCrA 1, ISO-ChaI 217 , and ISO-Oph 102. As the typical width of a BD outflow is not known but reasonable estimates of outflow velocity can be made from measured radial velocities, method B is more suitable

Table 4

Physical Conditions in the Outflows of LS-RCrA 1 and ISO-ChaI 217 as Defined by $n_{e}, x_{e}, T_{e}$, and $n_{H}$

\begin{tabular}{cccccc}
\hline \hline Object & Slit P.A. $\left(^{\circ}\right)$ & $n_{e}\left(\mathrm{~cm}^{-3}\right)$ & $x_{e}$ & $T_{e}\left(10^{3} \mathrm{~K}\right)$ & $n_{H}\left(10^{5} \mathrm{~cm}^{-3}\right)$ \\
\hline LS-RCrA 1 & 45 & $1478( \pm 170)$ & $0.012( \pm 0.005)$ & $35( \pm 1)$ & $1.2( \pm 0.7)$ \\
& 63 & $1300( \pm 140)$ & $0.004( \pm 0.002)$ & $37( \pm 2)$ & $3.3( \pm 2.0)$ \\
& 74 & $1300( \pm 120)$ & $0.002( \pm 0.001)$ & $41( \pm 1)$ & $6.7( \pm 2.3)$ \\
& 82 & $1500( \pm 200)$ & $0.002( \pm 0.001)$ & $44( \pm 3)$ & $8.6( \pm 2.7)$ \\
\hline ISO-ChaI 217 & $0 \mathrm{~B}$ & $1900( \pm 700)$ & $\ldots$ & $\ldots$ & $\ldots$ \\
& $0 \mathrm{R}$ & $5700( \pm 2300)$ & $\ldots$ & $\ldots$ & $\ldots$ \\
& $90 \mathrm{~B}$ & $600( \pm 200)$ & $\ldots$ & $\ldots$ & $\ldots$ \\
\hline
\end{tabular}

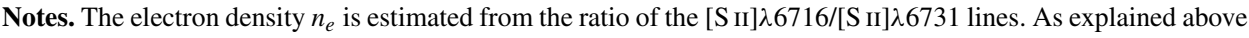
$x_{e}, T_{e}$, and $n_{H}$ could only be measured in the case of LS-RCrA 1 and this was done using the BE technique. While estimates of $n_{e}$ are reasonable when compared to measurements made for low-mass protostellar outflows, values of $x_{e}$ are far less and $T_{e}, n_{H}$ far greater than would be expected. Several factors make it currently very difficult to applying techniques such as the BE technique to BD spectra (refer to Section 4.4).

Table 5

Values Used in the Calculation of $\dot{M}_{\text {out }}$ from the Method of Hartigan et al. (1995)

\begin{tabular}{lcccc}
\hline \hline \multicolumn{1}{c}{ Variable } & LS-RCrA 1 & ISO-ChaI 217 (red) & ISO-ChaI 217 (blue) & ISO-Oph 102 \\
\hline EW [O I] $]$ 6300 $(\AA)$ & -30.0 & $\ldots$ & $\ldots$ & -2 \\
EW [S II] $]$ 6731 $(\AA)$ & -6.4 & -0.7 & -0.3 & -0.2 \\
$R_{0}$ & $19.2^{\mathrm{a}}$ & 16.59 & 16.59 & 14.57 \\
$L\left[\mathrm{O}_{\mathrm{I}}\right] \lambda 6300\left(L_{\odot}\right)$ & $7.1 \times 10^{-7}$ & $\ldots$ & $\ldots$ & $3.1 \times 10^{-6}$ \\
$L\left[\mathrm{~S} \mathrm{II} \lambda_{6731}\left(L_{\odot}\right)\right.$ & $1.4 \times 10^{-7}$ & $2.1 \times 10^{-7}$ & $9.1 \times 10^{-8}$ & $3.1 \times 10^{-7}$ \\
$n_{e}\left(\mathrm{~cm}^{-3}\right)$ & 1300 & 3900 & 1250 & $10^{3}-10^{4}$ \\
$i\left(^{\circ}\right)$ & 30 & 20 & 20 & 30 \\
$V_{\tan }\left(\mathrm{km} \mathrm{s}^{-1}\right)$ & 43 & 55 & 110 & 78 \\
$D(\mathrm{pc})$ & 130 & 140 & 140 & 125 \\
$l_{\tan }{ }^{\prime \prime}$ & 1.2 & 1 & 1 & 1 \\
\hline
\end{tabular}

Notes. As both lobes of the ISO-ChaI 217 outflow are not resolved spectrally in the [O I] 6300 line it was not possible to measure their separate EWs so no values are given here.

${ }^{\text {a }} R_{0}$ is the dereddened magnitude and for LS-RCrA 1 is taken from Comerón et al. (2003). 
Table 6

Measurements of $\dot{M}_{\text {out }}$ and $\dot{M}_{\text {acc }}$ for the Objects Studied To Date

\begin{tabular}{|c|c|c|}
\hline Object & $\dot{M}_{\text {out }}\left(M_{\odot} \mathrm{yr}^{-1}\right)$ & Method \\
\hline \multicolumn{3}{|l|}{ LS-RCrA 1} \\
\hline & $2.4 \times 10^{-9}$ & A \\
\hline & $6.1 \times 10^{-10}$ & $\mathrm{~B}[\mathrm{O} \mathrm{I}] \lambda 6300$ \\
\hline & $2.0 \times 10^{-10}$ & B [S II] $\lambda 6731$ \\
\hline \multicolumn{3}{|l|}{ ISO-Oph 102} \\
\hline & $1.7-11.8 \times 10^{-10}$ & B $[S$ II $] \lambda 6731$ \\
\hline & $1.4 \times 10^{-9}$ & CO molecular outflow ${ }^{1}$ \\
\hline \multicolumn{3}{|c|}{ ISO-ChaI 217 Red flow } \\
\hline & $3.1 \times 10^{-10}$ & B [S II] $] 26731$ \\
\hline \multicolumn{3}{|c|}{ ISO-ChaI 217 Blue flow } \\
\hline & $1.8 \times 10^{-10}$ & B [S II] $] 6731$ \\
\hline \multicolumn{3}{|l|}{ LS-RCrA 1} \\
\hline & $2.8 \times 10^{-10}$ & $\mathrm{CaII}(\lambda 8542)^{2}$ \\
\hline & $10^{-10}-10^{-9}$ & Optical Veiling ${ }^{3}$ \\
\hline & $10^{-9}$ & $\mathrm{CaII}(\lambda 8662)^{4}$ \\
\hline & $10^{-10}$ & $\mathrm{H} \alpha 10 \%$ width $^{5}$ \\
\hline \multicolumn{3}{|l|}{ ISO-Oph 102} \\
\hline & $10^{-9}$ & $\mathrm{H} \alpha 10 \%$ width $^{6}$ \\
\hline & $4.3 \times 10^{-10}$ & $J$ and $K$ band spectra ${ }^{7}$ \\
\hline \multicolumn{3}{|l|}{ ISO-ChaI 217} \\
\hline & $1.0 \times 10^{-10}$ & $\mathrm{H} \alpha$ emission $^{8}$ \\
\hline
\end{tabular}

Notes. For the BDs in our sample $\dot{M}_{\text {out }}$ is comparable to $\dot{M}_{\text {acc }} .1=$ Phan-Bao et al. (2008), 2 = Comerón et al. (2003), 3 = Barrado y Navascués et al. (2004), $4=$ Mohanty et al. (2005), $5=$ Scholz \& Jayawardhana (2006), $6=$ Natta et al. (2004), 7 = Natta et al. (2006), $8=$ Muzerolle et al. (2005).

at present as a tool for estimating $\dot{M}_{\text {out }}$. An added advantage of method B is that Equation (4) is less sensitive to $n_{e}$ which is useful for cases where $n_{e}$ is not known and the [S II] 66731 line alone is detected. Method B has allowed us to limit $\dot{M}_{\text {out }}$ for all three objects to the range $10^{-10}$ to $10^{-9} M_{\odot} \mathrm{yr}^{-1}$. Quantities such as jet velocity and electron density must be better constrained in order to tighten this range. While the measured range for $\dot{M}_{\text {acc }}$ is at present equally as large and is of order a few times $10^{-10}$ $M_{\odot} \mathrm{yr}^{-1}$ up to of order $10^{-9}$, what can be concluded here is that $\dot{M}_{\text {out }}$ for the BDs in our sample is comparable to $\dot{M}_{\text {acc }}$. However, some important points to note are as follows. The number of objects in our sample is very small and the standard $\dot{M}_{\text {acc }}$ versus $\dot{M}_{\text {out }}$ correlation for CTTSs shows a very wide scatter either side of the fitted least squares straight line (Hartigan et al. 1995). Importantly, there may also be some selection effects in our sample. As our observations are difficult and at the edge of what the VLT can do (the [O I] 66300 line emission is only just observable), one would therefore tend to pick up only objects with higher outflow rates (brighter [O I] emission) all else being equal. In other words, in the plot (for CTTSs) mentioned above we might be selecting only the brighter (upper) points in the scatter.

Difficulties related to measuring $\dot{M}_{\text {acc }}$ in BDs should also be considered. The most common methods for deriving $\dot{M}_{\text {acc }}$ are based on modeling of lines like $\mathrm{H} \alpha$ or $\mathrm{Pa} \beta$ (Natta et al. 2004), on veiling measurements and on the flux of Ca II lines like the CaII $\lambda 8662$ (Mohanty et al. 2005). However, accretion rate estimates for BDs based on different lines (e.g., Halpha or $\mathrm{Ca} \mathrm{H} \& \mathrm{~K}$ ) give very different results (Mohanty et al. 2005). Individual lines (e.g., $\mathrm{H} \alpha$ ) vary on timescales of weeks (Scholz et al. 2005; Nguyen et al. 2009) and thus the rates measured at a particular epoch may not reflect the long-term accretion rate that is more appropriate for comparison with $\dot{M}_{\text {out }}$. A second conceivable difficulty with these approaches is posed by any possible contribution to the $\mathrm{H} \alpha$ or $\mathrm{Ca}$ II lines from an outflow. For example, Whelan et al. (2009) measure a significant contribution to the LS-RCrA 1 outflow in the wings of the $\mathrm{H} \alpha$ line. Overall, further investigation is needed to confirm if in BDs a larger fraction of infalling material is re-ejected in an outflow than in low-mass stars. One theory of BD formation is that they originate from cores that never accrete enough material to enter an H-burning phase. A mass outflow rate which is a significant fraction of the infall rate could account for this. Future work on measuring $\dot{M}_{\text {out }}$ for young BD outflows should focus on increasing the number of objects known to have outflows, on obtaining better quality spectra and on imaging studies to allow outflow width and velocity to be derived. This work would also benefit from expansion into the NIR regime (Podio et al. 2006).

\section{CLASSICAL T TAURI-LIKE OUTFLOW ACTIVITY IN THE BROWN DWARF MASS REGIME}

Much of what is now known about the launching of protostellar jets comes from investigating the FEL regions of CTTSs. The first long-slit spectroscopic studies allowed their morphology, kinematics, and P.A. to be studied (Hirth et al. 1997) and progress in this area has been driven by high angular resolution techniques (Ray et al. 2007). A notable feature of the FEL regions of CTTSs is that they often have two velocity components, a low velocity component (LVC) believed to trace a wide disk wind, and a high velocity component (HVC) tracing the jet. Currently, the scale over which jets are collimated, the jet rotation, the jet excitation conditions, and mass outflow rate are all estimated from their FEL regions (Ray et al. 2007). While the distinctive FEL profiles associated with outflow activity were first studied in the spectra of CTTSs (Hirth et al. 1997), Class I low-mass protostars and Herbig Ae/Be stars are now also known to show strong FE. Studies have compared the FEL regions of the above objects to those of the CTTSs (Boehm \& Catala 1994; Corcoran \& Ray 1998; Davis et al. 2003; Garcia Lopez et al. 2008). The earliest studies which identified FEL tracers of mass outflow in the spectra of BDs include Fernández \& Comerón (2001) and Barrado y Navascués et al. (2004). Whelan et al. (2007) presented evidence which supported the hypothesis that BD outflows are scaled-down from CTT outflows. Here, recent new evidence is summarized. As all the BDs investigated to date are optically visible it is assumed that they are the substellar mass counterparts to the CTTSs i.e., equivalent to Class II low-mass sources.

For the five BDs whose FEL spectra have been investigated to date the $[\mathrm{O}$ I] $\lambda 6300$ line is the dominant line. The $[\mathrm{S} \mathrm{II}] \lambda 6731$ line is detected in three cases (LS-RCrA 1, ISO-ChaI 217 and ISO-Oph 102) and only for LS-RCrA 1 is the $[\mathrm{N} \mathrm{II]}] \lambda 6583$ line detected at a reasonable $\mathrm{S} / \mathrm{N}$. Very faint $[\mathrm{N}$ II $] \lambda 6583$ emission is found in the spectrum of ISO-ChaI 217. Interestingly, for LS-RCrA 1, Whelan et al. (2009) detected both the blue- and redshifted outflow in the wings of the $\mathrm{H} \alpha$ line. This was the first time, $\mathrm{H} \alpha$ was shown to originate in a $\mathrm{BD}$ outflow. As only blueshifted FE is found, this points to a dust hole in the LS-RCrA 1 disk, of an estimated size of $\sim 7$ AU (refer to Whelan et al 2009 for further information). Spectro-astrometric studies of permitted emission lines have led to similar discoveries for the disk of CTTSs (Whelan et al. 2004; Takami et al. 2001). This is an important parallel between the disk-outflow systems of BDs and CTTSs.

A second striking parallel between outflow activity in BDs and CTTSs is offered by the emergence of evidence of a LVC to BD FELs. Again this comes from the recent study of LS-RCrA 1. 
Although the LVC and HVC are not resolved spectrally for the LS-RCrA 1 FELs, Whelan et al. (2009) argue that the large spread in radial velocity with the [OI] 26300 line having the lowest velocity $\left(-5 \mathrm{~km} \mathrm{~s}^{-1}\right)$ and the $[\mathrm{N} \mathrm{II}] \lambda 6583$ line the highest $\left(-22 \mathrm{~km} \mathrm{~s}^{-1}\right.$ with a wing extending to $\left.-75 \mathrm{~km} \mathrm{~s}^{-1}\right)$ makes it clear that the $[\mathrm{O} \mathrm{I}] \lambda 6300$ line is predominantly tracing a LVC component to the outflow and the [N II] $] 2583$ line the HVC. The [S II] 6731 line shows evidence of both a LVC and a HVC. In the case of ISO-Oph 32, the low radial velocity of the [O I] $\lambda 6300$ emission line is also suggestive of the presence of a LVC to the outflow driven by this BD. An explanation for the low velocity based on a edge-on disk scenario is less likely given the lack of redshifted emission (the 2MASS1207-3932 FELs also have low radial velocities but both blue- and redshifted emissions are seen), hence it is likely that the [OI] 6300 line has a strong contribution from a LVC. The confirmation of a LVC to BD outflows would makes it very likely that many of the same processes active in the launching region of BDs and CTTSs and thus strongly links BD and protostellar outflows. However, a large sample of young BDs must be investigated to obtain this confirmation.

As asymmetries are frequently observed in CTT outflows a third compelling piece of evidence supporting the continuation of CTT-like outflow activity into the BD regime, is the asymmetrical nature of the ISO-ChaI 217 outflow. Hirth et al. (1994) first reported asymmetries in the jets driven by the CTTS RW Aur and DO Tau. For RW Aur, the brighter redshifted lobe has a radial velocity a factor of 2 slower than the blueshifted lobe. In the case of DO Tau, while the blueshifted lobe is the brightest it is slower than the redshifted flow by a factor of 2.3. In addition, the results of Hirth et al. (1994) reveal a difference in the electron density between both lobes of the flows. For RW Aur, $n_{e}$ is higher in the red lobe than the blue, however, for DO Tau the brighter lobe has the lowest electron density. Hirth et al. (1994) also list several other protostars for whom radial velocity asymmetries have been reported highlighting that this is a common feature of protostellar outflows. The discovery of such strong asymmetries so close to the driving source is important as it shows that these asymmetries are intrinsic to the central engine and suggests that their origin is in the jet launching mechanism itself. Ferreira et al. (2006) also discuss the origin of asymmetries in CTT jets. The asymmetries in the FELs of ISO-ChaI 217 are remarkably similar to what has been well studied for RW Aur. In the spectra taken at both $0^{\circ}$ and $90^{\circ}$, the radial velocity of the redshifted component is a factor of $\sim 2$ higher than that of the blueshifted lobe, the redshifted lobe is brighter, and initial results suggest that the electron density of the redshifted flow is also higher. Spectra taken along the actual outflow P.A. (estimated here at $\sim 200^{\circ}$ ) would allow a more detailed investigation of the ISO-ChaI 217 outflow asymmetries.

Finally, we consider the existing evidence that BD outflows also have a molecular component. Although early observations of CTT jets concentrated on the optical regime observations now extend into the near-infrared (Davis et al. 2003; Beck et al. 2008). Takami et al. (2004) first reported a detection of a warm molecular component to the DG Tau jet (observed in the $\mathrm{H}_{2}$, $v=1-0 \mathrm{~S}(1)$ line) and observations of $\mathrm{H}_{2}$ in the spectra of CTTSs are now not unusual. Note however that shocked $\mathrm{H}_{2}$ emission in outflows is much more common where a source is in the embedded phase, i.e., Class 0-I (Davis et al. 2001). While there has been very little investigation of BD outflow activity in the NIR, LS-RCrA 1 is known to show strong $\mathrm{H}_{2}$ emission at $2.122 \mu \mathrm{m}$ (Fernández \& Comerón 2001). A recent significant discovery in the field of BD outflows is the observation of a CO $J=2-1$ molecular outflow driven by ISO-Oph 102 (Phan-Bao et al. 2008). ISO-Oph 102 was the first BD shown by us to drive an outflow (Whelan et al. 2005). Phan-Bao et al. (2008) used the sub-millimeter array (SMA) to observe a small-scale molecular outflow driven by ISO-Oph 102. The total scale of the outflow is $\sim 20^{\prime \prime}$ and it is aligned at a N-S P.A. CTTSs are optically visible and therefore no longer embedded in their natal material. It is argued that molecular outflows originate as circumstellar material is entrained and driven outwards by an underlying jet (Downes \& Cabrit 2007), therefore the most spectacular protostellar molecular outflows are driven by embedded protostars such as Class 0 or Class I low-mass stars (Arce et al. 2007). CO outflows are driven by the youngest CTTSs (Launhardt et al. 2009) and observations are rare. The existence of a molecular component to BD outflows is a fourth noteworthy similarity between BD and low-mass protostellar outflows.

\section{SUMMARY}

Currently, our understanding of astrophysical outflows is focused on observations of low-mass protostellar objects. Although much can be gleaned from such observations a proper appreciation of the outflow phenomenon can only be reached through a complete investigation of how outflow activity compares for a range of driving sources, varying in mass and age. The new results included in this paper include the presentation of two new BD outflows, the unusual redshifted asymmetry in the ISO-ChaI 217 and the demonstration that $\dot{M}_{\text {out }}$ in BDs is comparable to $\dot{M}_{\text {acc }}$. Overall, the main aim of our BD project is to explore how substellar outflow activity compares to what is well documented for low-mass protostars. As our sources are all optically visible our comparison is focussed on the CTTSs. A second advantage of this work is that it may add to the debate on the formation mechanism of BDs, i.e., whether they form like low-mass protostars or are ejected embryos from multiple systems. The later mechanism is thought to produce truncated disks and very little envelope. If BD outflows are long-lived, this would argue against such a scenario although further investigation is required.

Due to the faintness of the BD outflows observations are difficult and time consuming. However, data gathered to date has led to some significant results. The most compelling pieces of evidence which support the continuation of CTT-like outflow activity into the $\mathrm{BD}$ mass regime include, the detection of an outflow component to the LS-RCrA $1 \mathrm{H} \alpha$ emission line and the suggestion of a possible disk dust hole, the identification of an LVC to the FELs of LS-RCrA 1 and ISO-Oph 32, the strong asymmetry in the ISO-ChaI 217 outflow and the discovery of a molecular component to the optical outflows of LS-RCrA 1 and ISO-Oph 102.

While these results are significant, much further work is needed to provide a more complete picture of how BD and CTT outflows compare. This is especially true for any study of the physical conditions in BD outflows and BD mass outflow rates. A considerably increased sample is needed. This can be provided through further optical and NIR spectroscopic studies complemented by millimeter studies. Investigations must be extended to other wavelength regimes so that NIR atomic and molecular outflow tracers can be identified and further $\mathrm{CO}$ outflows found. Finally, optical/NIR images would allow the collimation of the BD outflows to be probed. Many of these projects are underway or planned however due to faintness of 
the emission from a typical BD outflows, it is likely that longslit/echelle spectroscopy combined with spectro-astrometry will remain (in the short-term at least) the most effective way to investigate BD outflow activity.

The authors thank A. Natta for her interest in this project and her very valuable comments on this paper. E.T.W. is supported by an IRCSET-Marie Curie International Mobility Fellowships in Science, Engineering, and Technology within the 7th European Community Framework Programme. The present work was supported in part by the European Community's Marie Curie Actions-Human Resource and Mobility within the JETSET (Jet Simulations, Experiments, and Theory) network under contract MRTN-CT-2004 005592).

\section{REFERENCES}

Andre, P., Ward-Thompson, D., \& Barsony, M. 1993, ApJ, 406, 122

Apai, D., Pascucci, I., Bouwman, J., Natta, A., Henning, T., \& Dullemond, C. P. 2005, Science, 310, 834

Arce, H. G., Shepherd, D., Gueth, F., Lee, C.-F., Bachiller, R., Rosen, A., \& Beuther, H. 2007, in Protostars and Planets V, Molecular Outflows in Lowand High-Mass Star-forming Regions, ed. B. Reipurth, D. Jewitt, \& K. Keil (Tucson, AZ: Univ. Arizona), 245

Bacciotti, F., \& Eislöffel, J. 1999, A\&A, 342, 717

Bacciotti, F., Eislöffel, J., \& Ray, T. P. 1999, A\&A, 350, 917

Barrado y Navascués, D., Mohanty, S., \& Jayawardhana, R. 2004, ApJ, 604, 284

Bary, J. S., Weintraub, D. A., Shukla, S. J., Leisenring, J. M., \& Kastner, J. H. 2008, ApJ, 678, 1088

Beck, T. L., McGregor, P. J., Takami, M., \& Pyo, T.-S. 2008, ApJ, 676, 472

Boehm, T., \& Catala, C. 1994, A\&A, 290, 167

Camenzind, M. 2005, Mem. Soc. Astron. Ital., 76, 98

Comerón, F., Fernández, M., Baraffe, I., Neuhäuser, R., \& Kaas, A. A 2003, A\&A, 406, 1001

Corcoran, M., \& Ray, T. P. 1998, A\&A, 331, 147

Davis, C. J., Ray, T. P., Desroches, L., \& Aspin, C. 2001, MNRAS, 326, 524

Davis, C. J., Whelan, E., Ray, T. P., \& Chrysostomou, A. 2003, A\&A, 397, 693

Dekker, H., D’Odorico, S., Kaufer, A., Delabre, B., \& Kotzlowski, H. 2000, Proc. SPIE, 4008, 534

Dougados, C., Cabrit, S., Lavalley, C., \& Ménard, F. 2000, A\&A, 357, L61

Downes, T. P., \& Cabrit, S. 2007, A\&A, 471, 873

Fendt, C., \& Zinnecker, H. 1998, A\&A, 334, 750

Fernández, M., \& Comerón, F. 2001, A\&A, 380, 264

Fernández, M., \& Comerón, F. 2005, A\&A, 440, 1119

Ferreira, J., Dougados, C., \& Cabrit, S. 2006, A\&A, 453, 785

Garcia Lopez, R., Nisini, B., Giannini, T., Eislöffel, J., Bacciotti, F., \& Podio, L. 2008, A\&A, 487, 1019

Gatti, T., Testi, L., Natta, A., Randich, S., \& Muzerolle, J. 2006, A\&A, 460, 547

Hartigan, P., Edwards, S., \& Ghandour, L. 1995, ApJ, 452, 736

Hartigan, P., Morse, J. A., \& Raymond, J. 1994, ApJ, 436, 125

Herczeg, G. J., Cruz, K. L., \& Hillenbrand, L. A. 2009, ApJ, 696, 1589

Hirth, G. A., Mundt, R., \& Solf, J. 1997, A\&AS, 126, 437

Hirth, G. A., Mundt, R., Solf, J., \& Ray, T. P. 1994, ApJ, 427, L99

James, D. J., Melo, C., Santos, N. C., \& Bouvier, J. 2006, A\&A, 446, 971

Jayawardhana, R., Ardila, D. R., Stelzer, B., \& Haisch, K. E., Jr. 2003a, AJ, 126,1515
Jayawardhana, R., Mohanty, S., \& Basri, G. 2003b, ApJ, 592, 282

Lada, C. J. 1987, in Proc. Symp. on Star Forming Regions, Vol. 115, ed. M. Peimbert \& J. Jugaku (Dordrecht: D. Reidel), 1

Launhardt, R., et al. 2009, A\&A, 494, 147

López-Martín, L., Cabrit, S., \& Dougados, C. 2003, A\&A, 405, L1

López Martí, B., Eislöffel, J., Scholz, A., \& Mundt, R. 2004, A\&A, 416, 555

Luhman, K. L. 2004, ApJ, 602, 816

Luhman, K. L., Rieke, G. H., Young, E. T., Cotera, A. S., Chen, H., Rieke, M J., Schneider, G., \& Thompson, R. I. 2000, ApJ, 540, 1016

Martín, E. L., Delfosse, X., \& Guieu, S. 2004, AJ, 127, 449

Masciadri, E., \& Raga, A. C. 2004, ApJ, 615, 850

Mohanty, S., Jayawardhana, R., \& Basri, G. 2004, ApJ, 609, 885

Mohanty, S., Jayawardhana, R., \& Basri, G. 2005, ApJ, 626, 498

Mohanty, S., Jayawardhana, R., Huélamo, N., \& Mamajek, E. 2007, ApJ, 657, 1064

Mundt, R., \& Fried, J. W. 1983, ApJ, 274, L83

Muzerolle, J., Luhman, K. L., Briceño, C., Hartmann, L., \& Calvet, N. 2005, ApJ, 625, 906

Natta, A., Testi, L., Comerón, F., Oliva, E., D’Antona, F., Baffa, C., Comoretto, G., \& Gennari, S. 2002, A\&A, 393, 597

Natta, A., Testi, L., Muzerolle, J., Randich, S., Comerón, F., \& Persi, P. 2004, A\&A, 424, 603

Natta, A., Testi, L., \& Randich, S. 2006, A\&A, 452, 245

Nguyen, D. C., Scholz, A., van Kerkwijk, M. H., Jayawardhana, R., \& Brandeker, A. 2009, ApJ, 694, L153

Nisini, B., Bacciotti, F., Giannini, T., Massi, F., Eislöffel, J., Podio, L., \& Ray, T. P. 2005, A\&A, 441, 159

Osterbrock, D. E. 1994, Astrophysics of Gaseous Nebulae and Active Galactic Nuclei (Mill Valley, CA: Univ. Science Books)

Pascucci, I., Apai, D., Luhman, K., Henning, T., Bouwman, J., Meyer, M. R. Lahuis, F., \& Natta, A. 2009, ApJ, 696, 143

Persi, P., et al. 2000, A\&A, 357, 219

Phan-Bao, N., et al. 2008, ApJ, 689, L141

Podio, L., Bacciotti, F., Nisini, B., Eislöffel, J., Massi, F., Giannini, T., \& Ray, T. P. 2006, A\&A, 456, 189

Podio, L., Garcia, P. J. V., Bacciotti, F., Antoniucci, S., Nisini, B., Dougados, C., \& Takami, M. 2008, A\&A, 480, 421

Ray, T., Dougados, C., Bacciotti, F., Eislöffel, J., \& Chrysostomou, A. 2007, in Protostars and Planets V, Toward Resolving the Outflow Engine: An Observational Perspective, ed. B. Reipurth, D. Jewitt, \& K. Keil (Tucson, AZ: Univ. Arizona), 231

Reipurth, B., \& Bally, J. 2001, ARA\&A, 39, 403

Scholz, A., Jayawardhana, R., \& Brandeker, A. 2005, ApJ, 629, L41

Scholz, A., \& Jayawardhana, R. 2006, ApJ, 638, 1056

Solf, J., \& Boehm, K. H. 1993, ApJ, 410, L31

Stahler, S. W. 1983, ApJ, 274, 822

Takami, M., Bailey, J., Gledhill, T. M., Chrysostomou, A., \& Hough, J. H. 2001, MNRAS, 323, 177

Takami, M., Chrysostomou, A., Ray, T. P., Davis, C., Dent, W. R. F., Bailey, J., Tamura, M., \& Terada, H. 2004, A\&A, 416, 213

Whelan, E., \& Garcia, P. 2008, in Lecture Notes in Physics, Vol. 742, Jets from Young Stars II, ed. F. Bacciotti, E. T. Whelan, \& L. Testi (Berlin: Springer), 123

Whelan, E. T., Ray, T. P., Bacciotti, F., Natta, A., Testi, L., \& Randich, S. 2005, Nature, 435, 652

Whelan, E. T., Ray, T. P., \& Bacciotti, F. 2009, ApJ, 691, L106

Whelan, E. T., Ray, T. P., \& Davis, C. J. 2004, A\&A, 417, 247

Whelan, E. T., Ray, T. P., Randich, S., Bacciotti, F., Jayawardhana, R., Testi, L., Natta, A., \& Mohanty, S. 2007, ApJ, 659, L45

Woitas, J., Ray, T. P., Bacciotti, F., Davis, C. J., \& Eislöffel, J. 2002, ApJ, 580 336 


\title{
ERRATUM: “CLASSICAL T TAURI-LIKE OUTFLOW ACTIVITY IN THE BROWN DWARF MASS REGIME” (2009, ApJ, 706, 1054)*
}

\author{
E. T. Whelan ${ }^{1}$, T. P. Ray ${ }^{2}$, L. Podio ${ }^{3}$, F. Bacciotti ${ }^{3}$, And S. Randich ${ }^{3}$ \\ ${ }^{1}$ Institut für Astronomie und Astrophysik, Kepler Center for Astro and Particle Physics, Eberhard Karls Universität, D-72076 Tübingen, Germany \\ ${ }^{2}$ Dublin Institute for Advanced Studies, School of Cosmic Physics, 31 Fitzwilliam Place, Dublin 2, Ireland \\ ${ }^{3}$ INAF/Osservatorio Astrofisico di Arcetri, Largo Enrico Fermi 5, I-50125 Firenze, Italy \\ Received 2014 April 18; published 2014 June 6
}

Figures 3-11 have an error in that the direction of the offsets should be reversed. Thus, in the spectro-astrometric plots, positive offsets are toward the top of the figure and negative offsets toward the bottom. In the position-velocity diagram shown in Figure 8, positive offsets are toward the left and negative toward the right. The result of this correction is that the orientations of the blueshifted and redshifted lobes of the outflows are now reversed. Thus, for ISO-Cha1 217 the blueshifted flow is the in northeast quadrant with a position angle (P.A.) of $20^{\circ}$ and the redshifted flow is in the southwest quadrant (see Figure 2). Similarly, for ISO-Oph 32 , the blueshifted flow now lies in the northeast quadrant with a P.A. of $60^{\circ}$. Table 3 has been updated accordingly.

Table 3

Position Angles of the Two Outflows Observed

\begin{tabular}{lcc}
\hline \hline Object & Line & $\begin{array}{c}\text { Outflow P.A. } \\
\left({ }^{\circ}\right)\end{array}$ \\
\hline ISO-ChaI 217 & {$[\mathrm{O}$ I $] \lambda 6300$} & $29( \pm 8)$ \\
& {$[\mathrm{O}$ I $] \lambda 6363$} & $26( \pm 15)$ \\
{$[\mathrm{S}$ II $] \lambda 6716$} & $13( \pm 5)$ \\
& {$[\mathrm{S}$ I $] \lambda 6731$} & $20( \pm 6)$ \\
\hline ISO-Oph 32 & {$[\mathrm{O}$ I $] \lambda 6300$} & $60( \pm 7)$ \\
\hline
\end{tabular}

Notes. The P.A.s are estimated from orthogonal spectra as described in Section 3. For ISO-ChaI 217 a bipolar outflow is uncovered and estimates given above are for the blueshifted lobe. For ISO-Oph 32 only the blueshifted outflow is detected.

\footnotetext{
* Based on data collected by UVES observations (079.C-0375(A)) at the Very Large Telescope on Cerro Paranal (Chile) which is operated by the European Southern Observatory (ESO).
} 